\title{
$M^{a} \vee$ e phenomena

\section{Towards more general constitutive relations for metamaterials: a checklist for consistent formulations}

Fatima Z. Goffi, Karim Mnasri, Michael Plum, Carsten Rockstuhl, Andrii Khrabustovskyi

CRC Preprint 2019/24, December 2019

\section{KARLSRUHE INSTITUTE OF TECHNOLOGY}

\section{CRC 1173}

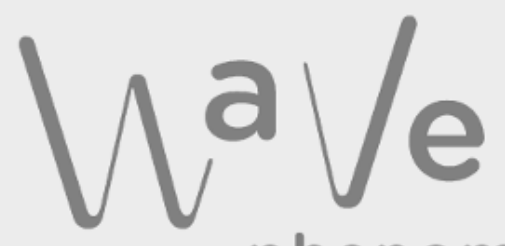

phenomena 


\section{Participating universities}

EBERHARD KARLS UNIVERSITATT TUBINGEN
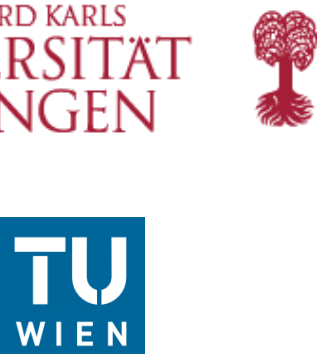

Funded by

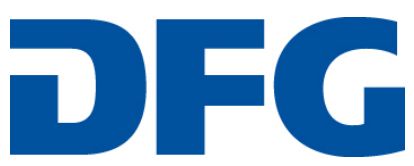




\title{
Towards more general constitutive relations for metamaterials: a checklist for consistent formulations
}

\author{
Fatima Z. Goffi ${ }^{*}, 1$, Karim Mnasri², Michael Plum ${ }^{1}$, Carsten Rockstuhl' ${ }^{2,3}$, and Andrii \\ Khrabustovskyi ${ }^{4,5}$ \\ ${ }^{1}$ Institute for Analysis, Karlsruhe Institute of Technology, Englerstraße 2, 76131 Karlsruhe, \\ Germany \\ ${ }^{2}$ Institute of Theoretical Solid State Physics, Karlsruhe Institute of Technology, \\ Wolfgang-Gaede-Str. 1, 76131 Karlsruhe, Germany \\ ${ }^{3}$ Institute of Nanotechnology, Karlsruhe Institute of Technology, P.O. Box 3640, 76021 \\ Karlsruhe, Germany \\ ${ }^{4}$ Department of Physics, Faculty of Science, University of Hradec Králové, Rokitanského 62, \\ 50003 Hradec Králové, Czech Republic \\ ${ }^{5}$ Institute of Applied Mathematics, Graz University of Technology, Steyrergasse 30, 8010 \\ Graz, Austria
}

\begin{abstract}
Abstract - When the period of unit-cells constituting metamaterials is no longer much smaller than the wavelength but only smaller, local material laws fail to describe the propagation of light in such composite media when considered at the effective level. Instead, nonlocal material laws are required. They have to be derived by approximating a general response function of the electric field in the metamaterial at the effective level that is accurate but cannot be handled practically. But how to perform this approximation is not obvious at all. Indeed many approximations can be perceived and one should be able to decide as quick as possible which of these possible material laws are mathematically and physically meaningful at all. Here, at the example of a second order Padé approximation of the general response function of the electric field, we present a checklist each possible constitutive relation has to pass in order to be physically and mathematically liable. As will be shown, only one out of these nine Padé approximations passes the checklist. The work is meant to be a guideline applicable to decide which constitutive relation makes actually sense at all. It is an essential ingredient for future research on composite media as any possible constitutive relation to be discussed should pass it.
\end{abstract}

\section{Introduction}

Electromagnetic metamaterials (MMs) are artificial structures made of subwavelength inclusions. These inclusions are called meta-atoms and they are mostly arranged in a periodic manner. The purpose of constructing MMs is to control the light propagation in a way inaccessible with natural materials. A referential purpose, but of course not the only one, would be to achieve a material where the real parts of both the permittivity and the permeability can be simultaneously negative at some frequencies. Because of their properties, MMs enable many applications. For instance, super lenses [1, 2], cloaking devices [25], medical devices [4], and many others (see, e.g., $[5,6,7,8,9]$ ) could be mentioned.

Due to the mesoscopic features of MMs, their homogenization was always a prime theoretical challenge. The homogenization consists of linking the mesoscopic structure of the actual MM to a macroscopic homogeneous material, i.e., replacing a MM by a hypothetical homogeneous material described by some effective material parameters [10]. Many homogenization techniques can be found in literature (see, e.g., [11, 14, 15, 16]). However, local constitutive relations have been, mostly, considered in the homogenization and it was frequently assumed

*Corresponding Author: fatima.goffi@kit.edu 
that the MM possesses a weak spatial dispersion (WSD). An effective description with at most bi-anisotropic constitutive relations would be the most advanced model to describe the MM at the level of such local constitutive relations [21]. Local constitutive relations are only justified as long as the period of the meta-atoms' arrangement is much smaller than the wavelength of light [22].

Unfortunately, most MMs do have a period only smaller than the wavelength and period and operational wavelength are actually often in the same order of magnitude. This implies that WSD is insufficient to capture the properties of most actual MMs. A natural extension to capture the physics of MMs at the effective level, therefore, are nonlocal constitutive relations [23, 24]. There, the induced response does not just depend on the electric and magnetic field at the same spatial locations but also on the fields at points further apart. Alternatively, the response depends besides on the fields also on the gradients of the field at the same location, a notion that is usually called strong spatial dispersion (SSD) [25]. We take both terms, nonlocality and SSD, as synonymous here. Constitutive relations accommodating SSD or nonlocality, respectively, were already discussed by several authors, see, e.g., [26, 27, 28, 30, 32, 33]. It was unambiguously shown that they frequently capture the properties of actual MMs much more accurately than ordinary local constitutive relations. But how to come to meaningful nonlocal constitutive relations for the effective description? It is clear that any description has to depart from a general nonlocal response function $\mathbf{R}\left(\omega, \mathbf{r}-\mathbf{r}^{\prime}\right.$ ) (also written in spatial Fourier space as $\widehat{\mathbf{R}}(\omega, \mathbf{k}))$ that expresses the response at the effective level as a convolution. Here, the response function links the electric field to the electric displacement. It constitutes an exact description but the use of this nonlocal response function at the effective level, however, is cumbersome and not handy. It has to be approximated if it shall be of any practical use. But how to approximate it in a suitable manner?

The general nonlocal response function can be expanded, as one possible path towards meaningful nonlocal constitutive relations, by a Taylor polynomial [30, 31]. Truncating the Taylor polynomial to the fourth order, forcing the coefficients of the Taylor polynomials to obey certain relations, and while omitting odd order terms that vanish for MMs with an inversion symmetry, as we do assume also here, the following constitutive relation can be derived:

$$
\widehat{\mathbf{D}}(\omega, \mathbf{k})=\varepsilon(\omega) \widehat{\mathbf{E}}(\omega, \mathbf{k})-\mathbf{k} \times(\alpha(\omega) \mathbf{k} \times \widehat{\mathbf{E}})(\omega, \mathbf{k})+\mathbf{k} \times \mathbf{k} \times(\gamma(\omega) \mathbf{k} \times \mathbf{k} \times) \widehat{\mathbf{E}}(\omega, \mathbf{k}) .
$$

In the real space, the corresponding expression to Eqn. (1) is given by

$$
\mathbf{D}(\omega, \mathbf{r})=\varepsilon(\omega) \mathbf{E}(\omega, \mathbf{r})+\nabla \times \alpha(\omega) \nabla \times \mathbf{E}(\omega, \mathbf{r})+\nabla \times \nabla \times(\gamma(\omega) \nabla \times \nabla \times) \mathbf{E}(\omega, \mathbf{r}) .
$$

The material parameters $\varepsilon(\omega), \alpha(\omega)$, and $\gamma(\omega)$ are anisotropic diagonal matrices, which implies that in general they do not commute with the curl operators. There is a physical meaning behind each of these terms. The first term corresponds to a local electric response, and indeed the permittivity $\varepsilon(\omega)$ appears here. It is a local response because the induced electric displacement field $\mathbf{D}(\omega, \mathbf{r})$ depends locally on the electric field $\mathbf{E}(\omega, \mathbf{r})$. The second term is associated with a weak spatial dispersion [34]. However, while it obviously depends on spatial derivatives of the electric field, a suitable gauge transformation to Maxwell's equations can be applied that forces the appearance of this second term as an artificial local magnetic response [36]. This implies that the magnetic field depends locally on the magnetic induction, where the response is mediated by the permeability that is explicitly expressed in terms of this parameter $\alpha(\omega)$. That finding is important as it shows that an artificial magnetism in MMs is a consequence of the weak spatial dispersion in the electric response. The third term in Eqns. (1) and (2) is special and indeed constitutes the suggested extension to capture effects due to strong spatial dispersion [30]. It is a consequence of the fourth order term in the Taylor polynomial. This term cannot be transformed to emerge in some local constitutive relation and is truly nonlocal. The choice of the specific functional dependency of that term can be motivated by the apparent similarity to the term that was used to capture the artificial magnetism, i.e. the material parameter is sandwiched between an equal number of curl operators.

To make practical use of such constitutive relation, interface conditions need to be derived that connect the fields inside the MM to the fields in a medium adjacent to an interface. These interface conditions can be derived for a given constitutive relation using a weak formulation to Maxwell's equations (see, e.g., [37]). Essential to nonlocal materials laws is the appearance of multiple modes [35]. While in a local material and for a given polarization of the electromagnetic field, the dispersion relation says that for a given frequency and transverse wave vector component there is only a single forward and a single backward propagating mode, nonlocal constitutive relations lead to multiple solutions. Therefore, not just ordinary interface conditions are needed but some additional (see, e.g., [29, 38]).

However, the choice of the specific model for the constitutive relation based on a Taylor polynomial that led to Eqn. (1) leaves the impression as being somewhat arbitrary. Many other approximations to the general nonlocal response function could have been considered and could have been applied to the homogenization [39]. A decision concerning a most appropriate model for the homogenization is always the question of how well the homogeneous model can capture the response from an actual MM. But of course, prior any consideration it is of utmost importance to know which model for a constitutive relation is eligible at all. It is the purpose of this 


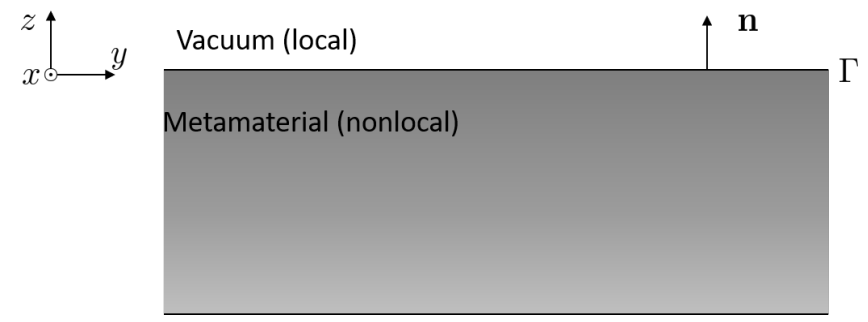

Figure 1: Illustration of the domain in which the light propagates, the upper-half space is occupied by vacuum and the lower-half space is occupied by a homogenized MM. The surface separating the two half-spaces is denoted $\Gamma$. The normal $\mathbf{n}$ is outward directed from the homogenized MM.

contribution to establish, at the example of a specific but systematic approach to generate nonlocal constitutive relations more complicated than those of the bi-anisotropic material, a checklist that can be used to validate whether a specific constitutive relation is admissible to homogenize a MM or not.

This suggested checklist has three entries. First, the dispersion relation of a nonlocal media, as mentioned, gives rise to multiple modes to be excited when an interface between an ordinary material and a nonlocal MM is illuminated with a plane wave. Therefore, we require to derive from the combination of constitutive relations and possible weak formulations the same number of interface conditions as modes supported in the MM. Neither more (overdetermined) nor less (underdetermined) interface conditions are admissible. Second, we require that the nonlocal models resort to the local models in the limiting case of a vanishing nonlocality. Third, reciprocity relations, expressed here in terms of the Casimir-Onsager relations, need to be fulfilled by the constitutive relations. As we show, many of the possible models one could imagine actually do not cope with these requirements and, indeed, expression (1) is already an excellent choice to express the fourth order term in the nonlocality.

We demonstrate the checklist, exemplary, while considering a more general expansion of the non-local response kernel, namely a Padé-approximation. The Padé-approximation has the advantage of expanding the nominator and the denominator or a rational function independently. Moreover, we can admit models where the material parameters are not written specifically between symmetric order of curl operators, as written in Eqn. (1). This offers a path to derive quite diverse nonlocal constitutive relations that may or may not capture the response from an actual MM quite well. However, before being considered for this task, these constitutive relations have to pass the suggested checklist.

This paper is organized as follows. In Sec. 2, we recall the Maxwell equations in the specific setting of interest. In Sec. 3, we talk about the concept of homogenization, by showing the difference between the local and nonlocal homogenization notions. In Sec. 4, we present constitutive relations for what we call Padé MMs obtained from a Padé-type approximation of the nonlocal response function. The nine possible cases to perform the Padé-type approximation constitute the constitutive relations expressing the properties of the MMs at the effective level. In Sec. 5, we give the solutions to the dispersion relations for the nine cases, by treating separately both transverse electric (TE) and transverse magnetic (TM) polarizations. For the sake of readability, a detailed derivation of the dispersion relations is presented in an Appendix. In Sec. 6, we set the function space we need in the present research, and we explain the essence of the weak formulation. Our main contribution is given in Sec. 7, in which we present the criteria we set for the checklist in order to analyze the validity of each case. These criteria are based on mathematical and physical first-principles. This necessarily requires also a derivation of the interface conditions that is done in Subsec. 7.1 as well. In Sec. 8, we discuss and summarize our findings. At the end of the paper we present in an appendix the detailed coefficients given in the solutions to the dispersion relations.

With this work, we unlock the opportunities to consider in the future other constitutive relations. Basically many formulations can be postulated but only those should be considered that pass the presented checklist.

\section{General setting}

For the sake of simplicity, we assume that the propagation of light takes place in the entire space $\mathbb{R}^{3}$, where the upper-half space $\mathbb{R}_{+}^{3}$ is occupied by vacuum and the lower-half space $\mathbb{R}_{-}^{3}$ is occupied by a MM. The interface between them is denoted by $\Gamma$. The unit normal $\mathbf{n}$ is outwardly directed from the homogeneous MM (see Fig. 1).

We recall Maxwell's equations with no external charges and currents, which govern the propagation of light 
in a material

$$
\begin{array}{ll}
\nabla \times \tilde{\mathbf{E}}+\frac{1}{c} \frac{\partial \tilde{\mathbf{B}}}{\partial t}=0, & \nabla \cdot \tilde{\mathbf{B}}=0 \\
\nabla \times \tilde{\mathbf{H}}-\frac{1}{c} \frac{\partial \tilde{\mathbf{D}}}{\partial t}=0, & \nabla \cdot \tilde{\mathbf{D}}=0 .
\end{array}
$$

This system of equations includes four vector fields: the electromagnetic field given by the pair $(\tilde{\mathbf{E}}, \tilde{\mathbf{H}})$ and the electromagnetic induction given by the pair $(\tilde{\mathbf{D}}, \tilde{\mathbf{B}})$. They depend on the position $\mathbf{r}$ and the time $t$ in $\mathbb{R}^{3} \times \mathbb{R} \longrightarrow \mathbb{C}^{3}$, but this dependency is omitted here for brevity. The speed of light is given by $c$ and the time frequency is given by $\omega>0$. For a harmonic time dependency, Maxwell's system is written as

$$
\begin{aligned}
\nabla \times \mathbf{E}-\mathrm{i} k_{0} \mathbf{B} & =0, & \nabla \cdot \mathbf{B} & =0, \\
\nabla \times \mathbf{H}+\mathrm{i} k_{0} \mathbf{D} & =0, & \nabla \cdot \mathbf{D} & =0 .
\end{aligned}
$$

Here, $k_{0}$ refers to the wavenumber of the external monochromatic light, it is given by the relation $k_{0}=\frac{\omega}{c}$. The electromagnetic MMs we are considering are supposed to be, only for simplicity, centro-symmetric, such that no optical activity emerges in the principal axes. A typical example of such structure is the Fishnet MM (see, e.g., [41, 42]). Providing the relation between the electromagnetic fields and the electromagnetic induction is required to solve the system of equation. These relations are the desired constitutive relation if considered at the effective level. For the mesoscopic, i.e. for the actual, MM, the usual local constitutive relations are considered and we assume that the MM itself is made from a non-magnetic media.

\section{Homogenization of metamaterials}

The modeling of phenomena occurring in composite materials leads generally to partial differential equations whose coefficients are strongly oscillating. In our case, these coefficients refer to the spatially dependent permittivity that describes the properties of the actual MM in real space. These oscillations can generate problems in the analytical and numerical resolution of these equations. Moreover, we do not wish to solve Maxwell's equations exactly anytime a device made from MMs is considered; but we wish to consider MMs actually on an equal footing as ordinary materials. The theory of homogenization serves to overcome these difficulties by replacing problems with strongly oscillating coefficients by approximate problems whose coefficients are constant, and therefore much simpler to process numerically. For an introduction to this theory we refer the reader to see for instance $[43,44,45]$. In the case of MMs, the homogenization process has to be done in order to associate the MM to its effective properties. The principle consists in defining a hypothetical homogeneous material, which is characterized by effective properties. We require that in the homogeneous material: (a) the propagation of light has to be analogous as in the actual MM and (b) the reflection and transmission coefficients on an interface, separating a MM to an adjacent known material, have to be also analogous for both the homogeneous material and the actual MM. For the description of MMs at the effective level, either local or nonlocal constitutive relations can be used.

\subsection{Local homogenization of metamaterials}

Most frequently, local constitutive relations are considered to homogenize MMs, where the electromagnetic field and the electromagnetic induction are linked to each other through local constitutive relations. This is a mere extension of how we treat natural materials in Maxwell's equations. The functional dependency of $\mathbf{D}$ and $\mathbf{H}$ is a linear combination of the macroscopic fields $\mathbf{E}$ and $\mathbf{B}$, that read

$$
\begin{aligned}
& \mathbf{D}(\omega, \mathbf{r})=\mathbf{E}(\omega, \mathbf{r})+\mathbf{P}(\mathbf{E})(\omega, \mathbf{r}), \\
& \mathbf{H}(\omega, \mathbf{r})=\mathbf{B}(\omega, \mathbf{r})-\mathbf{M}(\mathbf{B})(\omega, \mathbf{r}),
\end{aligned}
$$

where $\mathbf{P}$ is the polarization and $\mathbf{M}$ is the magnetization. They shall explicitly depend only on $\mathbf{E}$ and $\mathbf{B}$ at the same spatial location and also not on derivatives. Please note, for the centro-symmetric materials as considered here there is no electromagnetic cross-coupling, i.e. $\mathbf{P}$ does not depend on $\mathbf{B}$ and $\mathbf{M}$ does not depend on $\mathbf{E}$. For intrinsically non-magnetic materials, we have $\mathbf{M} \equiv 0$. To apply such constitutive relations, the size of the meta-atoms constituting the MM has to be much smaller than the wavelength, something that does not apply to most MMs frequently considered especially at optical frequencies. Most MMs do have a period only smaller than the wavelength and both of them are in the same order of magnitude. This implies that the WSD is insufficient to capture the properties of most actual MMs, and going beyond local homogenization with WSD is then needed for a proper homogenization.

A typical example of the local homogenization is the asymptotic homogenization (see, e.g., [15, 16, 17, 18, 19, 20]). The concept considers a small parameter $\delta$ referring to the period of the meta-atoms' arrangement. 
Homogenizing the Maxwell system consists in studying the asymptotic behavior of its solution when $\delta$ tends to 0 .

\subsection{Nonlocal homogenization of metamaterials}

To further advance the effective description, nonlocal constitutive relations with SSD are required. We consider the general nonlocal material law of a homogeneous medium written in the real space in the following form

$$
\mathbf{D}(\omega, \mathbf{r})=\int_{\mathbb{R}^{3}} \mathbf{R}\left(\omega, \mathbf{r}-\mathbf{r}^{\prime}\right) \mathbf{E}\left(\omega, \mathbf{r}^{\prime}\right) \mathrm{d} \mathbf{r}^{\prime}
$$

where the kernel $\mathbf{R}(\omega, \mathbf{r}-\cdot)$ represents the response function of the electric Field $\mathbf{E}$. If the response tensor $\mathbf{R}(\omega, \mathbf{r}-\cdot)$ contains distributional terms, the nonlocal material law (6) can be written as a dual pairing, i.e., as a distributional action, as follows

$$
\mathbf{D}(\omega, \mathbf{r})=\langle\mathbf{R}(\omega, \mathbf{r}-\cdot), \mathbf{E}(\omega, \cdot)\rangle .
$$

In general, the formula of the response tensor is unknown, hence it is not clear how to evaluate the electromagnetic field on the interface separating two disparate media. Finally, while the expression is certainly correct, it is unhandy for any practical purpose. Therefore, this expression of the response kernel needs to be simplified in order to reach a constitutive relation of practical utility. How this can be done will be discussed in the next section.

\section{Padé metamaterials}

\subsection{Constitutive relations}

In the spatial frequency space the nonlocal material law (6) is written in the following form

$$
\widehat{\mathbf{D}}(\omega, \mathbf{k})=\widehat{\mathbf{R}}(\omega, \mathbf{k}) \widehat{\mathbf{E}}(\omega, \mathbf{k}) .
$$

In [30] a specific approximation of the nonlocal response function based on a Taylor approximation up to the fourth order was considered. In this paper, we consider rational response functions, i.e., of Padé-type, written as

$$
\widehat{\mathbf{R}}(\omega, \mathbf{k})=(I-\widehat{\mathbf{q}}(\omega, \mathbf{k}))^{-1}(\varepsilon(\omega) I+\widehat{\mathbf{p}}(\omega, \mathbf{k})),
$$

where $I$ refers to the identity matrix, $\widehat{\mathbf{p}}(\omega, \cdot)$ and $\widehat{\mathbf{q}}(\omega, \cdot)$ are $\mathbb{C}^{3 \times 3}$ matrices, polynomially depending on $\mathbf{k}$ and vanish at $\mathbf{k}=0$. They are assumed to take the following expressions

$$
\begin{aligned}
& \widehat{\mathbf{p}}(\omega, \mathbf{k}) \in\{(-\alpha(\omega) \mathbf{k} \times \mathbf{k} \times),(-\mathbf{k} \times \alpha(\omega) \mathbf{k} \times),(-\mathbf{k} \times \mathbf{k} \times \alpha(\omega))\}, \\
& \widehat{\mathbf{q}}(\omega, \mathbf{k}) \in\{(-\gamma(\omega) \mathbf{k} \times \mathbf{k} \times),(-\mathbf{k} \times \gamma(\omega) \mathbf{k} \times),(-\mathbf{k} \times \mathbf{k} \times \gamma(\omega))\},
\end{aligned}
$$

with $\alpha(\omega)$ and $\gamma(\omega)$ representing the material parameters corresponding to the polynomials $\widehat{\mathbf{p}}$ and $\widehat{\mathbf{q}}$, respectively. Together with the electric permittivity $\varepsilon(\omega)$, they are assumed to be $\mathbb{C}^{3 \times 3}$ anisotropic diagonal matrices with smooth and bounded entries depending on the frequency $\omega$.

After backward Fourier transform, we see that the polynomial-matrices $\widehat{\mathbf{p}}$ and $\widehat{\mathbf{q}}$ amount to one of the following differential operators

$$
\begin{aligned}
& \mathbf{p}(\omega, \mathrm{i} \nabla) \in\{(\alpha(\omega) \nabla \times \nabla \times),(\nabla \times \alpha(\omega) \nabla \times),(\nabla \times \nabla \times \alpha(\omega))\}, \\
& \mathbf{q}(\omega, \mathrm{i} \nabla) \in\{(\gamma(\omega) \nabla \times \nabla \times),(\nabla \times \gamma(\omega) \nabla \times),(\nabla \times \nabla \times \gamma(\omega))\} .
\end{aligned}
$$

Thereafter, the nonlocal material law amounts to

$$
(I-\mathbf{q}(\omega, \mathrm{i} \nabla)) \mathbf{D}(\omega, \mathbf{r})=(\varepsilon(\omega) I+\mathbf{p}(\omega, \mathrm{i} \nabla)) \mathbf{E}(\omega, \mathbf{r}),
$$

which is a partial differential equation. According to the choices of $\mathbf{p}(\omega, \mathrm{i} \nabla)$ and $\mathbf{q}(\omega, \mathrm{i} \nabla)$, Eqn. (12) leads to nine different formulations that we call in the following "cases".

Let us now justify the assumptions on the material parameter of being anisotropic diagonal matrices. The anisotropy of a given material is the property of being directionally dependent. More precisely, anisotropic materials admit different properties in different directions. They can be represented by 9 element tensors, i.e., $\mathbb{C}^{3 \times 3}$ matrices. However, for a suitable choice of coordinate system, we can only have nonzero diagonal elements in the material parameters tensors, whereas all the off-diagonal elements are zero. For this reason, instead of full anisotropic tensors, we consider only diagonal matrices that simplify computations. Furthermore, if the three values for the diagonal elements are equal, we say that the material is isotropic. Under an isotropy assumption, 
the material parameters are simply scalars. This fact implies that the three different formulae of the operator $\mathbf{p}(\omega, \mathrm{i} \nabla)$ expressed in (11a) will be identical; the same will hold also for the operator $\mathbf{q}(\omega, \mathrm{i} \nabla)$ expressed in (11b). Hence, we get only one wave-like equation instead of several formulations.

In this paper, we adopted the naming Padé metamatrials because we used a Padé-type expansion for the nonlocal response function of the electric field inside MMs. Such kind of expansions, i.e., of Padé-type, were already considered in the literature, for example:

- when expressing the properties of metals within a hydrodynamic Drude-model, e.g. applicable to study for plasmonic nanostructures (see for example [47, 48]), where the nonlocal response function is given by

$$
\widehat{\mathbf{R}}(\omega, \mathbf{k})_{\text {Hydro }}=1-\frac{\omega_{p}^{2}}{\omega^{2}+\mathrm{i} \omega \gamma-\beta^{2}|\mathbf{k}|^{2}},
$$

for $\omega_{p}$ being the plasma frequency of the free electrons, $\gamma$ the damping constant, and $\beta$ a nonlocal term proportional to the Fermi velocity.

- when expressing the properties of wire media [49, 50], which constitutes an example of complex artificial electromagnetic materials, consisting of metallic nanowires embedded in a dielectric host medium. See for instance [51], where a nonlocal homogenization theory was applied to homogenize a wire medium and it was compared to a homogenization theory specifically derived for the wire medium in [52]. We can see clearly in [52] that the permittivity of a nanowire medium is of Padé-type, having the following form

$$
\varepsilon_{\mathrm{m}}=\varepsilon_{\text {bulk }}+\frac{\mathrm{i} \omega_{p}^{2} \tau\left(R_{b}-R\right)}{\omega(\omega \tau+\mathrm{i})\left(\omega \tau R+\mathrm{i} R_{b}\right)} .
$$

The first term of the permittivity, denoted by $\varepsilon_{\text {bulk }}$, corresponds to the permittivity of bulk gold. The second term represents additional effects, with $\omega_{p}$ being the plasma frequency, $R_{b}$ and $\mathrm{R}$ refer to the mean-free path and the effective mean-free path of the electrons respectively, $\tau$ refers to the relaxation time of the conducting electrons.

In the next subsection, we express one wave equation that governs the propagation of light in a medium characterized by each of these constitutive relations.

\subsection{Wave equations}

We recall Maxwell's equation for the electric field

$$
\nabla \times \nabla \times \mathbf{E}(\omega, \mathbf{r})=k_{0}^{2} \mathbf{D}(\omega, \mathbf{r})
$$

By substituting the general nonlocal material law (12) in the wave Eqn. (15), we obtain

$$
(I-\mathbf{q}(\omega, \mathrm{i} \nabla))(\nabla \times \nabla \times \mathbf{E}(\omega, \mathbf{r}))=k_{0}^{2}(\varepsilon(\omega) I+\mathbf{p}(\omega, \mathrm{i} \nabla)) \mathbf{E}(\omega, \mathbf{r}) .
$$

Hence, the general wave-like equation for homogenized MMs is given by

$$
\nabla \times \nabla \times \mathbf{E}(\omega, \mathbf{r})=k_{0}^{2} \varepsilon(\omega) \mathbf{E}(\omega, \mathbf{r})+\left[k_{0}^{2} \mathbf{p}(\omega, \mathrm{i} \nabla)+\mathbf{q}(\omega, \mathrm{i} \nabla)(\nabla \times \nabla \times)\right] \mathbf{E}(\omega, \mathbf{r}) .
$$

By combining each possible expression for $\mathbf{p}(\omega, \mathrm{i} \nabla)$ with each possible expression for $\mathbf{q}(\omega, \mathrm{i} \nabla)$, given in $(11 \mathrm{a})$ and (11b), we get nine equations modeling the propagation of light in a homogeneous MM. In a domain with upperhalf space vacuum and lower-half space MM, the wave-like equations generated from the Padé-type response functions, named from Case 1 to Case 9, are given in Tab. 1

Under the anisotropy assumption, the material parameters are $\mathbb{C}^{3 \times 3}$ diagonal matrices given by

$$
\tilde{\varepsilon}=\left(\begin{array}{ccc}
\tilde{\varepsilon}_{x x} & 0 & 0 \\
0 & \tilde{\varepsilon}_{y y} & 0 \\
0 & 0 & \tilde{\varepsilon}_{z z}
\end{array}\right), \tilde{\alpha}=\left(\begin{array}{ccc}
\tilde{\alpha}_{x x} & 0 & 0 \\
0 & \tilde{\alpha}_{y y} & 0 \\
0 & 0 & \tilde{\alpha}_{z z}
\end{array}\right), \tilde{\gamma}=\left(\begin{array}{ccc}
\tilde{\gamma}_{x x} & 0 & 0 \\
0 & \tilde{\gamma}_{y y} & 0 \\
0 & 0 & \tilde{\gamma}_{z z}
\end{array}\right),
$$

such that, for $j \in\{x, y, z\}$ we have

$$
\tilde{\varepsilon}_{j j}=\left\{\begin{array}{l}
1, \quad z>0, \\
\varepsilon_{j j}, \quad z<0,
\end{array} \quad \tilde{\alpha}_{j j}=\left\{\begin{array}{l}
0, \quad z>0, \\
\alpha_{j j}, \quad z<0,
\end{array} \quad \tilde{\gamma}_{j j}=\left\{\begin{array}{l}
0, \quad z>0 \\
\gamma_{j j}, \quad z<0
\end{array}\right.\right.\right.
$$

We stress that the second order terms in all equations in Tab. 1 describe the local effect, i.e., the effect produced using local constitutive relations. These terms can be regrouped by means of the parameter $\tilde{\mu}=\left(1-k_{0}^{2} \tilde{\alpha}\right)^{-1}$. We notice that for the formulae corresponding to cases 1, 2, and 3 in Tab. 1, the parameter $\tilde{\mu}$ represents the 


\begin{tabular}{|c|c|c|}
\hline $\begin{array}{l}\frac{\text { Case 1: }}{\nabla \times \nabla \times \mathbf{E}=} \\
k_{0}^{2} \tilde{E} \mathbf{E}+k_{0}^{2} \nabla \times \tilde{\alpha} \nabla \times \mathbf{E} \\
+\nabla \times \nabla \times \tilde{\gamma} \nabla \times \nabla \times \mathbf{E}\end{array}$ & $\begin{array}{l}\frac{\text { Case 2: }}{\nabla \times \nabla \times \mathbf{E}=} \\
k_{0}^{2} \tilde{\varepsilon} \mathbf{E}+k_{0}^{2} \nabla \times \tilde{\alpha} \nabla \times \mathbf{E} \\
+\nabla \times \tilde{\gamma} \nabla \times \nabla \times \nabla \times \mathbf{E}\end{array}$ & $\begin{array}{l}\frac{\text { Case 3: }}{\nabla \times \nabla \times \mathbf{E}=} \\
k_{0}^{2} \tilde{\varepsilon} \mathbf{E}+k_{0}^{2} \nabla \times \tilde{\alpha} \nabla \times \mathbf{E} \\
+\tilde{\gamma} \nabla \times \nabla \times \nabla \times \nabla \times \mathbf{E}\end{array}$ \\
\hline $\begin{array}{l}\text { Case 4: } \\
\nabla \times \nabla \times \mathbf{E}= \\
k_{0}^{2} \tilde{\varepsilon} \mathbf{E}+k_{0}^{2} \nabla \times \nabla \times \tilde{\alpha} \mathbf{E} \\
+\nabla \times \nabla \times \tilde{\gamma} \nabla \times \nabla \times \mathbf{E}\end{array}$ & $\begin{array}{l}\text { Case 5: } \\
\nabla \times \nabla \times \mathbf{E}= \\
k_{0}^{2} \tilde{\varepsilon} \mathbf{E}+k_{0}^{2} \nabla \times \nabla \times \tilde{\alpha} \mathbf{E} \\
+\nabla \times \tilde{\gamma} \nabla \times \nabla \times \nabla \times \mathbf{E}\end{array}$ & $\begin{array}{l}\text { Case 6: } \\
\nabla \times \nabla \times \mathbf{E}= \\
k_{0}^{2} \tilde{\varepsilon} \mathbf{E}+k_{0}^{2} \nabla \times \nabla \times \tilde{\alpha} \mathbf{E} \\
+\tilde{\gamma} \nabla \times \nabla \times \nabla \times \nabla \times \mathbf{E}\end{array}$ \\
\hline $\begin{array}{l}\text { Case 7: } \\
\nabla \times \nabla \times \mathbf{E}= \\
k_{0}^{2} \tilde{\varepsilon} \mathbf{E}+k_{0}^{2} \tilde{\alpha} \nabla \times \nabla \times \mathbf{E} \\
+\nabla \times \nabla \times \tilde{\gamma} \nabla \times \nabla \times \mathbf{E}\end{array}$ & $\begin{array}{l}\text { Case 8: } \\
\nabla \times \nabla \times \mathbf{E}= \\
k_{0}^{2} \tilde{\varepsilon} \mathbf{E}+k_{0}^{2} \tilde{\alpha} \nabla \times \nabla \times \mathbf{E} \\
+\nabla \times \tilde{\gamma} \nabla \times \nabla \times \nabla \times \mathbf{E}\end{array}$ & $\begin{array}{l}\text { Case 9: } \\
\nabla \times \nabla \times \mathbf{E}= \\
k_{0}^{2} \tilde{\varepsilon} \mathbf{E}+k_{0}^{2} \tilde{\alpha} \nabla \times \nabla \times \mathbf{E} \\
+\tilde{\gamma} \nabla \times \nabla \times \nabla \times \nabla \times \mathbf{E}\end{array}$ \\
\hline
\end{tabular}

Table 1: Wave-like equations modeling the propagation of light in media, where the upper-half space is vacuum and the lower-half space is a MM. These equations are obtained by means of a Padé-type expansion of the nonlocal response function to an incident electric field.

magnetic permeability. For the other cases, we don't have an explicit physical explanation. The nonlocal effect is given through the fourth order derivative terms, and it is represented by the parameter $\tilde{\gamma}$.

We emphasize that the present approach, i.e., Padé-type expansion of the response function, can be considered as a mathematical justification to the different locations of the material parameters expressed in Tab. 1. In other words, this approach shows that these models are not randomly chosen. Notably, due to the anisotropy of the material parameters, they do not commute with the curl operators. Together with the fact of approximating the nonlocal response function $\mathbf{R}$ by means of fractional functions, it is clear that we can easily end up at a large number of formulations. We highlight that possibly not all of these formulations are useful. Therefore, a checklist needs to be established, that allows to conclude which formulations are correct and which are not. Certainly, Padé-type approximation is not the only way for generating different formulations, and any future suggestions for a more general constitutive relation should respect such sort of checklist to end up with meaningful and sound results.

We remark that the first wave-like equation in Tab. 1, denoted as Case 1, coincides implicitly with the wave equation obtained in $[30,38,41]$ using a Taylor approximation of the response function truncated at the fourth order terms. Namely, for centro-symmetric MMs the constitutive relation in the real space is given by

$$
D_{i}(\omega, \mathbf{r})=a_{i j} E_{j}(\omega, \mathbf{r})+c_{i j l m} \nabla_{l} \nabla_{m} E_{j}(\omega, \mathbf{r})+e_{i j k l m n} \nabla_{k} \nabla_{l} \nabla_{m} \nabla_{n} E_{j}(\omega, \mathbf{r}) .
$$

The expansion coefficients are assumed to satisfy the following formulae

$$
\begin{aligned}
& a_{i j} \stackrel{!}{=} \varepsilon(\omega), \\
& c_{i j l m} \nabla_{l} \nabla_{m} E_{j} \stackrel{!}{=}[\nabla \times(\alpha(\omega) \nabla \times \mathbf{E})]_{i}, \\
& e_{i j k l m n} \nabla_{k} \nabla_{l} \nabla_{m} \nabla_{n} E_{j} \stackrel{!}{=}[\nabla \times \nabla \times(\gamma(\omega) \nabla \times \nabla \times \mathbf{E})]_{i} .
\end{aligned}
$$

Then, the obtained wave-like equation reads

$$
\nabla \times \nabla \times \mathbf{E}=k_{0}^{2}(\varepsilon(\omega) \mathbf{E}+\nabla \times \alpha(\omega) \nabla \times \mathbf{E}+\nabla \times \nabla \times \gamma(\omega) \nabla \times \nabla \times \mathbf{E}) .
$$

On the other hand, if we choose the operators $\mathbf{p}(\omega, i \nabla)$ and $\mathbf{q}(\omega, i \nabla)$ as follows

$$
\begin{aligned}
& \mathbf{p}(\omega, \mathrm{i} \nabla)=\nabla \times \alpha(\omega) \nabla \times, \\
& \mathbf{q}(\omega, \mathrm{i} \nabla)=\nabla \times \nabla \times \gamma(\omega),
\end{aligned}
$$

and we scale the parameter $\gamma(\omega)$, we get exactly the same equation as in case 1 of the Padé approximation. This equation was studied in detail in references [30,38, 41], and it provided good results (reflection and transmission coefficients) when compared to those obtained from a direct solution of Maxwell's equations for the actual MM. (see e.g., [53, 54]). Our main concern in this paper is to study the other cases in Tab. 1 and to compare them to case 1 .

Before presenting the established checklist, analyzing the wave-like equations obtained in the present paper, and solving the interface problems; we solve first the bulk problems. Namely, we compute and solve the dispersion relations which are required to define the propagating eigenmodes within a MM. 


\section{$5 \quad$ Dispersion relations}

For a plane wave propagating within a medium, the dispersion relation has to be satisfied to guarantee that the plane wave indeed solves Maxwell's equations [55]. The dispersion relation expresses the functional dependency of the wave vector components of the wave and its frequency for a given medium, i.e., $k_{z}\left(k_{x}, k_{y}, k_{0}, \varepsilon, \alpha, \gamma\right)$. For a homogeneous material, we can obtain the dispersion relation by plugging a plane wave ansatz in the wave equation. Hence, we get an algebraic system of equations instead of differential equations. For nontrivial solutions, the determinant of the corresponding matrix has to be zero. It is a polynomial with indeterminants the wave vector's components. The eigenvalues are the roots of the dispersion relation and the associated eigenmodes are the modes sustained inside the homogeneous material. In Fourier space, the general wave-like Eqn. (17) is written as

$$
\underbrace{\left[k_{0}^{2}(\varepsilon(\omega) I+\widehat{\mathbf{p}}(\omega, \mathbf{k}))+(I-\widehat{\mathbf{q}}(\omega, \mathbf{k}))(\mathbf{k} \times \mathbf{k} \times)\right]}_{\mathcal{W}(\omega, \mathbf{k})} \widehat{\mathbf{E}}(\omega, \mathbf{k})=0,
$$

for $\widehat{\mathbf{p}}(\omega, \mathbf{k})$ and $\widehat{\mathbf{q}}(\omega, \mathbf{k})$ having one of the forms expressed in (10a) and (10b). The vanishing of the determinant of the wave operator $\mathcal{W}(\omega, \mathbf{k})$ represents the dispersion relation. In vacuum, the dispersion relation is given by $k_{z}^{2}=k_{0}^{2}-\left(k_{x}^{2}+k_{y}^{2}\right)$. It has two solutions $k_{z}= \pm \sqrt{k_{0}^{2}-\left(k_{x}^{2}+k_{y}^{2}\right)}$ which represent the forward and the backward modes, respectively. In a bulk MM described by the effective material parameters (18), we will see that the dispersion relations are polynomials of order four, which gives rise to multiple, exactly four, modes propagating per polarization. Precisely, in the presence of interfaces, for example a MM slab with finite thickness, there will be two forward modes excited at the first interface and two backward modes at the second interface, instead of only one at each interface in the absence of strong spatial dispersion.

Without loss of generality, we assume that the incident wave propagates in the $y z$-plane with the $z$ direction as a principal propagation direction. Hence we set $k_{x}=0$ and the wave vector is written $\mathbf{k}=\left(0, k_{y}, k_{z}\right)^{T}$. Furthermore, along an interface $\Gamma$, the system is invariant under translation. Consequently, the wave vectors for all plane waves at the interface share the same $y$-component that we commonly denote by $k_{y}$. Further, we decompose the incident wave into its transverse electric (TE) and transverse magnetic (TM) polarized modes. Due to the absence of optical activity in centro-symmetric MMs, polarization is preserved for the reflected and transmitted fields, such that:

- TE-polarization:

$$
\mathbf{E}=\mathbf{E}_{0} \exp (\mathrm{ik} \cdot \mathbf{r}), \text { with } \quad \mathbf{E}_{0}=\left(E_{x}, 0,0\right)^{T} \text { and } \mathbf{k}=\left(0, k_{y}, k_{z}\right)^{T},
$$

- TM-polarization:

$$
\mathbf{E}=\mathbf{E}_{0} \exp (\mathrm{ik} \cdot \mathbf{r}), \text { with } \quad \mathbf{E}_{0}=\left(0, E_{y}, E_{z}\right)^{T} \text { and } \mathbf{k}=\left(0, k_{y}, k_{z}\right)^{T} .
$$

In what follows, we treat the TE and TM polarizations separately, i.e., we plug the corresponding polarized plane wave into the wave-like equation for each case in Tab. 1 and seek for the solutions $k_{z}\left(k_{x}, k_{y}, k_{0}, \varepsilon, \alpha, \gamma\right)$. In all nine cases the dispersion relations are polynomials of order 4 that depend on the polarization of the field. The corresponding solutions specify the forward and backward propagating eigenmodes inside such MMs. The solutions to the wave equation for the nine studied cases are summarized in Tab. 2. In fact, only five different functional dependencies of $k_{z}\left(k_{y}\right)$ emerge in these $2 \times 9$ resulting dispersion relations, leading to identical isofrequency contours. These five types are

Dispersion of type A: $k_{z}^{2}\left(k_{y}\right)=-\frac{1}{2}\left(q_{0}+q_{1}\right) k_{y}^{2}+p_{0} \pm \sqrt{l_{0}+\left(p_{0}+\frac{q_{0}-q_{1}}{2} k_{y}^{2}\right)^{2}}$,

Dispersion of type B: $k_{z}^{2}\left(k_{y}\right)=-k_{y}^{2}+p_{0} \pm \sqrt{l_{0}+p_{0}^{2}+2\left(p_{1}-p_{0}\right) k_{y}^{2}}$,

Dispersion of type C: $\quad k_{z}^{2}\left(k_{y}\right)=-\frac{1}{2}\left(q_{0}+q_{1}\right)+p_{0} \pm \sqrt{l_{0}+\left(p_{0}-\frac{q_{0}+q_{1}}{2} k_{y}^{2}\right)^{2}+2 q_{0} p_{1} k_{y}^{2}-q_{0} q_{1} k_{y}^{4}}$,

Dispersion of type D: $k_{z}^{2}\left(k_{y}\right)=-k_{y}^{2}+p_{0} \pm \sqrt{l_{0}+p_{0}^{2}}$, 


\begin{tabular}{|c|c|c|c|c|c|}
\hline $\mathbf{p}(\omega, \mathbf{k}) \quad \mathbf{q}(\omega, \mathbf{k})$ & $\mathbf{k} \times \mathbf{k} \times \gamma$ & \multicolumn{2}{|l|}{$\mathbf{k} \times \gamma \mathbf{k} \times$} & \multicolumn{2}{|l|}{$\gamma \mathbf{k} \times \mathbf{k} \times$} \\
\hline \multirow{2}{*}{$\mathbf{k} \times \alpha \mathbf{k} \times$} & Case 1 & \multicolumn{2}{|l|}{ Case 2} & \multicolumn{2}{|l|}{ Case 3} \\
\hline & TM: A & TM: A & TE: C & TM: C & TE: $B$ \\
\hline \multirow[t]{2}{*}{$\mathbf{k} \times \mathbf{k} \times \alpha$} & Case 4 & \multicolumn{2}{|l|}{ Case 5} & \multicolumn{2}{|l|}{ Case 6} \\
\hline & TM: C & TM: C & TE: A & TM: E & TE: D \\
\hline \multirow[t]{2}{*}{$\alpha \mathbf{k} \times \mathbf{k} \times$} & Case 7 & \multicolumn{2}{|l|}{ Case 8} & \multicolumn{2}{|l|}{ Case 9} \\
\hline & TM: C & TM: C & TE: A & TM: C & TE: D \\
\hline
\end{tabular}

Table 2: Summary of the different types of dispersion relations for the wave-like equations defined in Tab. 1, with respect to the transverse electric (TE) and the transverse magnetic (TM) polarized modes.

Dispersion of type E: $\quad Q_{0}\left(k_{y}\right) k_{z}^{2}\left(k_{y}\right)=P_{0}\left(k_{y}\right) \pm \sqrt{\left[P_{0}\left(k_{y}^{2}\right)\right]^{2}+P_{1}\left(k_{y}^{2}\right)}$.

Here, the coefficients $p_{0}, p_{1}, q_{0}, q_{1}, l_{0}, P_{0}, P_{1}$, and $Q_{0}$ are products and ratios of the material parameters and depend on the polarization and on the case. For the sake of readability, we summarize the exact coefficients of the dispersion relations in Appendix.

Concerning the first case, the dispersion relations (later the interface conditions as well) for both TE and TM polarization are identical to the wave equation considered by the Taylor approach that has been previously introduced in [30], with a normalization of the parameter expressing the nonlocal effects. The reader can return to $[38,30,41]$ for detailed discussions. Here, we repeat this dispersion relation for completeness. While eight out of nine Padé approximants yield a polynomial type of dispersion relations, the TM polarization of case 6 seems to be of special type, namely type $\mathrm{E}$. It is the only case, where the functional dependency of $k_{z}\left(k_{y}\right)$ is of Padé type as well, leading to peculiar isofrequency contours, inaccessible with the other eight cases. The above types of dispersion relations repeat also in a few occasions. The same type of dispersion relations will eventually reproduce the same bulk properties. In cases $(4,7)$ and $(5,8)$ the dispersion relations as well as their corresponding coefficients are pairwise identical. However, this does not forcibly mean that the corresponding pairs of cases are equivalent, as their interface conditions differ. Consequently, light at the interface will couple differently, leading to different electromagnetic response, i.e., reflection and transmission coefficients. To reach to these interfaces, we need to say something more general on the function space setting and the possible weak formulations that we discuss in the next section.

\section{$6 \quad$ Function spaces setting and weak derivatives}

The wave-like equations expressed in Tab. 1 are strong formulations for Padé MMs. $\mathbf{C}^{4}\left(\mathbb{R}^{3}\right)$-solutions ${ }^{1}$ do not exist. However, due to the discontinuity of $\tilde{\varepsilon}(\omega), \tilde{\alpha}(\omega)$, and $\tilde{\gamma}(\omega)$ on the surface $\Gamma$ separating the upper-half space vacuum to the lower-half space MM (see Fig. 1), the derivatives in Tab. 1 are no more considered as classical derivatives. Namely, these equations are understood in the generalized sense (see, e.g., [58]) and their solutions are generalized solutions, which are linear functionals acting continuously on smooth compactly supported functions. The space of generalized functions ${ }^{2}$ is denoted $\mathcal{D}^{\prime}\left(\mathbb{R}^{3}\right)$. This space represents the dual space of compactly supported smooth functions, i.e., $\mathbf{C}_{0}^{\infty}\left(\mathbb{R}^{3}\right)$.

Locally integrable functions on $\mathbb{R}^{3}$ (i.e., functions $f \in L_{\mathrm{loc}}^{1}\left(\mathbb{R}^{3}\right)$ ) provide generalized functions $F_{f}$, called regular generalized functions, acting on $\Psi \in C_{0}^{\infty}\left(\mathbb{R}^{3}\right)$ by

$$
F_{f}[\Psi]:=\int_{\mathbb{R}^{3}} f(\mathbf{r}) \Psi(\mathbf{r}) d \mathbf{r} .
$$

In the same manner, we can define the associated generalized functions for a subset of $L_{\text {loc }}^{1}\left(\mathbb{R}^{3}\right)$, which is the space of locally square integrable functions, i.e., $f \in L_{\text {loc }}^{2}\left(\mathbb{R}^{3}\right)$. In this paper, we deal with functions located in this space.

To solve the interface problems corresponding to equations in Tab. 1, we need to write them in a weaker formulation. The essence of this operation consists of formally multiplying the strong form ${ }^{3}$ with a test function, i.e., compactly supported smooth functions, and integrating over the domain on which the equations are defined. In the generalized sense, the definition of generalized derivatives mimics partial integration. For example, for $\mathbf{E} \in \mathbf{L}_{\text {loc }}^{2}\left(\mathbb{R}^{3}\right), \nabla \times \mathbf{E} \in \mathcal{D}^{\prime}\left(\mathbb{R}^{3}\right)$, and

$$
(\nabla \times \mathbf{E})[\mathbf{\Phi}]:=\int_{\mathbb{R}^{3}} \mathbf{E} \cdot(\nabla \times \mathbf{\Phi}) d \mathbf{r}, \quad \forall \mathbf{\Phi} \in \mathbf{C}_{0}^{\infty}\left(\mathbb{R}^{3}\right)
$$

\footnotetext{
${ }^{1} \mathbf{C}^{4}\left(\mathbb{R}^{3}\right)$ : the space of vector-functions, with components functions continuously differentiable up to order 4 .

${ }^{2}$ In some references they are called distributions (see, e.g., [58]).

${ }^{3}$ The governing partial differential equation, in which the order of derivatives is not reduced as in the weak form.
} 
Let us now consider a term appearing in cases 4,5 , and 6 in Tab. 1 , given by $\nabla \times \nabla \times \tilde{\alpha} \mathbf{E}$, and show how to define the weak derivation. For $\mathbf{F}:=\tilde{\alpha} \mathbf{E} \in \mathbf{L}_{\text {loc }}^{2}\left(\mathbb{R}^{3}\right)$ and $\nabla \times \nabla \times \tilde{\alpha} \mathbf{E} \in \mathcal{D}^{\prime}\left(\mathbb{R}^{3}\right)$, by definition we have

$$
(\nabla \times \nabla \times \mathbf{F})[\mathbf{\Phi}]:=\int_{\mathbb{R}^{3}} \mathbf{F} \cdot(\nabla \times \nabla \times \mathbf{\Phi}) d \mathbf{r}, \quad \forall \mathbf{\Phi} \in \mathbf{C}_{0}^{\infty}\left(\mathbb{R}^{3}\right)
$$

On the other hand, we have

$$
\int_{\mathbb{R}^{3}} \mathbf{F} \cdot(\nabla \times \nabla \times \mathbf{\Phi}) d \mathbf{r}=\int_{\mathbb{R}^{3}} \tilde{\alpha} \mathbf{E} \cdot(\nabla \times \nabla \times \mathbf{\Phi}) d \mathbf{r}=\int_{\mathbb{R}_{-}^{3}} \alpha \mathbf{E} \cdot(\nabla \times \nabla \times \boldsymbol{\Phi}) d \mathbf{r} .
$$

Thus, the weak derivative of $\nabla \times \nabla \times \tilde{\alpha} \mathbf{E}$ reads

$$
(\nabla \times \nabla \times \mathbf{F})[\mathbf{\Phi}]=\int_{\mathbb{R}_{-}^{3}} \alpha \mathbf{E} \cdot(\nabla \times \nabla \times \mathbf{\Phi}) d \mathbf{r}, \quad \forall \mathbf{\Phi} \in \mathbf{C}_{0}^{\infty}\left(\mathbb{R}^{3}\right) .
$$

According to the same principle, we define the weak derivatives in all the wave-like equations in Tab. 1 (see Sec. 7).

Always dealing with the term $\nabla \times \nabla \times \tilde{\alpha} \mathbf{E}$, we stress that due to the discontinuity of $\tilde{\alpha} \mathbf{E}$ at the surface $\Gamma$, it is not correct to consider the following weak derivative

$$
(\nabla \times \nabla \times \mathbf{F})[\mathbf{\Phi}]=\int_{\mathbb{R}_{-}^{3}}(\nabla \times \alpha \mathbf{E}) \cdot(\nabla \times \mathbf{\Phi}) d \mathbf{r}, \quad \forall \mathbf{\Phi} \in \mathbf{C}_{0}^{\infty}\left(\mathbb{R}^{3}\right),
$$

because it is not possible to define the corresponding regular generalized function to $\nabla \times \mathbf{F}$ since $\nabla \times \mathbf{F}=$ $\nabla \times \tilde{\alpha} \mathbf{E} \notin \mathbf{L}_{\text {loc }}^{1}\left(\mathbb{R}^{3}\right)$.

After explaining the principle of generalized derivatives and weak formulation, we solve the interface problems for the different cases presented in Tab. 1 by deriving the interface conditions. For the sake of shortness, we combine this task with the checklist for each model in Tab. 1 in the next section.

\section{Selection criteria and discussions}

To decide which case can possibly describe the propagation of light in homogeneous MMs, we shall present mathematical and physical criteria that intervene basically in the checking process.

- At first, we need to solve the interface problem for each case, which reveals the main principle one has to respect in such study. We highlight that the number of unknown field amplitudes at the interface $\Gamma$ is defined through the dispersion relations by counting the number of eigenmodes (cf. Sec. 5). To define their amplitudes, the number of eigenmodes must coincide with the number of interface conditions that we can derive at the interface $\Gamma$.

- At second, as the nonlocal approach should always contain the local approach as a limit, we have to analyze the resulting reflection and transmission coefficients calculated from the Fresnel equations. To this end, we check the limit of these coefficients when $\gamma \rightarrow 0$. More precisely, they have to be the same as the reflection and transmission coefficients produced by the WSD approach. In case of mismatch, the case corresponding to the chosen weak formulation cannot be considered for further applications.

- The third criterion that must be fulfilled in the effective description of optical MMs is the conformity with the Casimir-Onsager reciprocity principle [56], which imposes some symmetry conditions for the nonlocal response function $\widehat{\mathbf{R}}(\omega, \mathbf{k})$.

In the following subsection we derive first of all the interface conditions for all nine cases and verify their conformity with our first criteria. Afterwards, we impose in following subsections the other criteria and rule out an increasing number of cases to possibly express constitutive relations.

\subsection{Interface conditions analysis}

The number of eigenmodes excited in a half-space or a slab MM is determined from the dispersion relations (cf. Sec. 5 and [29]). When spatial dispersion occurs, the classical interface conditions are not sufficient to compute the amplitudes of all eigenmodes. For every constitutive relation proposed in Tab. 1, light-matter interaction is modeled differently. Therefore, one has to derive additional interface conditions for each case separately. In the literature they are not often calculated analytically, but rather introduced on phenomenological grounds (see, 
e.g., [24]). For that reason, solving the interface problems by exploiting the weak formulation of each wave-like equation in Tab. 1 represents one of our main contributions in this investigation.

Before starting, we recall Green's formula for the $\nabla \times$-derivative (see, e.g., [40]). For $\Omega$ a bounded domain in $\mathbb{R}^{3}$ with a Lipschitz boundary $\partial \Omega=\Gamma$, we define the space $\mathbf{H}(\mathbf{c u r l}, \Omega)$ as follows

$$
\mathbf{H}(\mathbf{c u r l}, \Omega)=\left\{\mathbf{u} \in \mathbf{L}^{2}(\Omega), \nabla \times \mathbf{u} \in \mathbf{L}^{2}(\Omega)\right\} .
$$

For $\mathbf{u}$ and $\mathbf{v}$ two vector fields in the space $\mathbf{H}(\mathbf{c u r l}, \Omega)$, we have the following Green's formula

$$
\int_{\Omega} \mathbf{u} \cdot(\nabla \times \mathbf{v}) d \mathbf{r}=\int_{\Omega}(\nabla \times \mathbf{u}) \cdot \mathbf{v} d \mathbf{r}+\int_{\Gamma}(\mathbf{u} \times \mathbf{n}) \cdot \mathbf{v}_{t} d \mathbf{s},
$$

where $\mathbf{v}_{t}$ represents the tangential component of the vector field $\mathbf{v}$ on the surface $\Gamma$ and $d \mathbf{s}$ represents the area of the surface element on $\Gamma$. The normal vector $\mathbf{n}$ is outwardly directed from $\Omega$.

For $\mathbf{E}$ a vector field defined in the upper-half space $\mathbb{R}_{+}^{3}$, we denote by $\mathbf{E}_{+}$its trace on the surface $\Gamma$. Conversely, for $\mathbf{E}$ a vector field defined in the lower-half space $\mathbb{R}_{-}^{3}$, we denote by $\mathbf{E}_{-}$its trace on the surface $\Gamma$.

The strategy of deriving the interface conditions consists in writing the corresponding weak formulations of the wave-like equations. A weak formulation requires formally writing the equations in the function space $\mathcal{D}^{\prime}\left(\mathbb{R}^{3}\right)$ (cf. Sec. 6 ). Then, we can derive the additional interface conditions by re-establishing the strong formulations via partial integration.

We emphasize that because of reasons linked to the discontinuity of the material parameters on the surface $\Gamma$, it is not always possible to write symmetric weak formulations. Namely, the bilinear form associated to the respective weak formulation is not always symmetric, i.e., the order of derivatives applied on the vector field $\mathbf{E}$ is not always the same as the order of derivatives applied on the test function $\mathbf{\Phi}$. Actually, our plan is not necessarily shifting all the $\nabla \times$-derivatives located before the parameters $\alpha$ and $\gamma$ to the test function $\boldsymbol{\Phi}$.

Some cases will be already excluded due to violation of the first criterion. Regarding the other cases, they need to fulfill the second and the third criteria. As previously mentioned, case 1 coincides implicitly with the model given by the Taylor approach studied in [38], in this paper we will just recall its corresponding interface conditions for a self contained paper.

\subsubsection{Interface conditions for Case 1}

We recall the wave-like equation corresponding to case 1 :

$$
\nabla \times \nabla \times \mathbf{E}=k_{0}^{2} \tilde{\varepsilon} \mathbf{E}+k_{0}^{2} \nabla \times \tilde{\alpha} \nabla \times \mathbf{E}+\nabla \times \nabla \times \tilde{\gamma} \nabla \times \nabla \times \mathbf{E} .
$$

Weak formulation: Eqn. (26) is understood in the generalized sense. For $\mathbf{E}$ having the following regularities

$$
\mathbf{E} \in \mathbf{L}_{\text {loc }}^{2}\left(\mathbb{R}^{3}\right), \quad \nabla \times \mathbf{E} \in \mathbf{L}_{\text {loc }}^{2}\left(\mathbb{R}^{3}\right), \quad \text { and } \quad \nabla \times \nabla \times \mathbf{E} \in \mathbf{L}_{\text {loc }}^{2}\left(\mathbb{R}_{-}^{3}\right),
$$

and for $\boldsymbol{\Phi} \in \mathbf{C}_{0}^{\infty}\left(\mathbb{R}^{3}\right)$, we define the weak formulation corresponding to the Eqn. (26) as follows

$$
\begin{aligned}
\int_{\mathbb{R}^{3}}(\nabla \times \mathbf{E}) \cdot(\nabla \times \mathbf{\Phi}) d \mathbf{r}=k_{0}^{2} & \int_{\mathbb{R}_{+}^{3}} \mathbf{E} \cdot \mathbf{\Phi} d \mathbf{r}+k_{0}^{2} \int_{\mathbb{R}_{-}^{3}} \varepsilon \mathbf{E} \cdot \mathbf{\Phi} d \mathbf{r} \\
& +k_{0}^{2} \int_{\mathbb{R}_{-}^{3}}(\alpha \nabla \times \mathbf{E}) \cdot(\nabla \times \mathbf{\Phi}) d \mathbf{r}+\int_{\mathbb{R}_{-}^{3}}(\gamma \nabla \times \nabla \times \mathbf{E}) \cdot(\nabla \times \nabla \times \mathbf{\Phi}) d \mathbf{r}
\end{aligned}
$$

We say that $\mathbf{E}$ is a weak solution to the wave-like Eqn. (26) if it has the regularities (27) and satisfies the weak formulation (28).

Interface conditions: (See [38])

$$
\begin{aligned}
& \left(\mathbf{E}_{+}-\mathbf{E}_{-}\right) \times \mathbf{n}=0, \\
& \left(\nabla \times \mathbf{E}_{+}-\nabla \times \mathbf{E}_{-}\right) \times \mathbf{n}+k_{0}^{2}\left(\alpha \nabla \times \mathbf{E}_{-}\right) \times \mathbf{n}+\left(\nabla \times \gamma \nabla \times \nabla \times \mathbf{E}_{-}\right) \times \mathbf{n}=0, \\
& \left(\gamma \nabla \times \nabla \times \mathbf{E}_{-}\right) \times \mathbf{n}=0 .
\end{aligned}
$$

The third term in the second interface condition (29b) is purely coming from nonlocality. The interface condition (29c) represents the one what we call in this context "the additional interface condition". In contrast to Maxwell's equations with the classical constitutive relations, we only have two interface conditions given by

$$
\begin{aligned}
& \left(\mathbf{E}_{+}-\mathbf{E}_{-}\right) \times \mathbf{n}=0, \\
& \left(\nabla \times \mathbf{E}_{+}-\mu^{-1} \nabla \times \mathbf{E}_{-}\right) \times \mathbf{n}=0 .
\end{aligned}
$$

Here, $\mu$ represents the magnetic permeability, that can be written as follows $\mu=\left(1-k_{0}^{2} \alpha\right)^{-1}$ (cf. Subsec. 4.2$)$. 


\subsubsection{Analysis of Case 2}

The wave-like equation corresponding to case 2 reads

$$
\nabla \times \nabla \times \mathbf{E}=k_{0}^{2} \tilde{E} \mathbf{E}+k_{0}^{2} \nabla \times \tilde{\alpha} \nabla \times \mathbf{E}+\nabla \times \tilde{\gamma} \nabla \times \nabla \times \nabla \times \mathbf{E} .
$$

Weak formulation: In the generalized sense, for $\mathbf{E}$ verifying the following regularities

$$
\mathbf{E} \in \mathbf{L}_{\mathrm{loc}}^{2}\left(\mathbb{R}^{3}\right), \quad \nabla \times \mathbf{E} \in \mathbf{L}_{\mathrm{loc}}^{2}\left(\mathbb{R}^{3}\right), \quad \text { and } \quad \nabla \times \nabla \times \nabla \times \mathbf{E} \in \mathbf{L}_{\mathrm{loc}}^{2}\left(\mathbb{R}_{-}^{3}\right),
$$

and for $\mathbf{\Phi} \in \mathbf{C}_{0}^{\infty}\left(\mathbb{R}^{3}\right)$, the weak formulation of the wave-like Eqn. (31) reads

$$
\begin{aligned}
\int_{\mathbb{R}^{3}}(\nabla \times \mathbf{E}) \cdot(\nabla \times \mathbf{\Phi}) d \mathbf{r}=k_{0}^{2} & \int_{\mathbb{R}_{+}^{3}} \mathbf{E} \cdot \mathbf{\Phi} d \mathbf{r}+k_{0}^{2} \int_{\mathbb{R}_{-}^{3}} \varepsilon \mathbf{E} \cdot \mathbf{\Phi} d \mathbf{r} \\
& +k_{0}^{2} \int_{\mathbb{R}_{-}^{3}}(\alpha \nabla \times \mathbf{E}) \cdot(\nabla \times \mathbf{\Phi}) d \mathbf{r}+\int_{\mathbb{R}_{-}^{3}}(\gamma \nabla \times \nabla \times \nabla \times \mathbf{E}) \cdot(\nabla \times \mathbf{\Phi}) d \mathbf{r} .
\end{aligned}
$$

We say that $\mathbf{E}$ is a weak solution to the wave-like Eqn. (31) if it has the regularities (32) and satisfies the weak formulation (33).

\section{Interface conditions:}

First interface condition: It represents a natural interface condition obtained from the fact that $\nabla \times \mathbf{E} \in$ $\mathbf{L}_{\text {loc }}^{2}\left(\mathbb{R}^{3}\right)$ and not from the weak formulations. This is why it represents also the first interface condition for all the other cases. The proof will be presented only in this paragraph, and for the other cases we cite it without showing the proof again.

For $\mathbf{E} \in \mathbf{L}_{\text {loc }}^{2}\left(\mathbb{R}^{3}\right)$ and $\nabla \times \mathbf{E} \in \mathbf{L}_{\text {loc }}^{2}\left(\mathbb{R}^{3}\right)$, we have

$$
\forall \mathbf{\Phi} \in \mathbf{C}_{0}^{\infty}\left(\mathbb{R}^{3}\right): \int_{\mathbb{R}^{3}}(\nabla \times \mathbf{E}) \cdot \mathbf{\Phi} d \mathbf{r}=\int_{\mathbb{R}^{3}} \mathbf{E} \cdot(\nabla \times \mathbf{\Phi}) d \mathbf{r} .
$$

Integrating by parts on each half-space, we get

$$
\begin{aligned}
\int_{\mathbb{R}^{3}}(\nabla \times \mathbf{E}) \cdot \mathbf{\Phi} d \mathbf{r} & =\int_{\mathbb{R}_{+}^{3}}(\nabla \times \mathbf{E}) \cdot \mathbf{\Phi} d \mathbf{r}+\int_{\mathbb{R}_{-}^{3}}(\nabla \times \mathbf{E}) \cdot \mathbf{\Phi} d \mathbf{r} \\
& =\int_{\mathbb{R}_{+}^{3}} \mathbf{E} \cdot(\nabla \times \mathbf{\Phi}) d \mathbf{r}+\int_{\mathbb{R}_{-}^{3}} \mathbf{E} \cdot(\nabla \times \mathbf{\Phi}) d \mathbf{r}+\int_{\Gamma}\left(\mathbf{E}_{+} \times \mathbf{n}-\mathbf{E}_{-} \times \mathbf{n}\right) \cdot \boldsymbol{\Phi}_{t} d \mathbf{s} \\
& =\int_{\mathbb{R}^{3}} \mathbf{E} \cdot(\nabla \times \mathbf{\Phi}) d \mathbf{r}+\int_{\Gamma}\left(\mathbf{E}_{+} \times \mathbf{n}-\mathbf{E}_{-} \times \mathbf{n}\right) \cdot \mathbf{\Phi}_{t} d \mathbf{s}
\end{aligned}
$$

Since $\boldsymbol{\Phi}$ is arbitrary, we get from Eqns. (34) and (35) the first interface condition, given by

$$
\left(\mathbf{E}_{+}-\mathbf{E}_{-}\right) \times \mathbf{n}=[\mathbf{E} \times \mathbf{n}]=0,
$$

where $[\cdot]$ represents the jump across the surface $\Gamma$.

Second interface condition: For $\mathbf{E}$ a weak solution to (31) with the regularities $\mathbf{E} \in \mathbf{L}_{\text {loc }}^{2}\left(\mathbb{R}^{3}\right), \nabla \times \nabla \times \mathbf{E} \in$ $\mathbf{L}_{\text {loc }}^{2}\left(\mathbb{R}^{3}\right)$, by taking first a test function $\mathbf{\Phi} \in \mathbf{C}_{0}^{\infty}\left(\mathbb{R}_{+}^{3}\right)$ and then in $\mathbf{C}_{0}^{\infty}\left(\mathbb{R}_{-}^{3}\right)$, we can show that:

$$
\begin{aligned}
& \nabla \times \nabla \times \mathbf{E}=k_{0}^{2} \mathbf{E}, \quad \text { for a.e. } \mathrm{x} \in \mathbb{R}_{+}^{3}, \\
& \nabla \times \nabla \times \mathbf{E}=k_{0}^{2} \varepsilon \mathbf{E}+k_{0}^{2} \nabla \times \alpha \nabla \times \mathbf{E}+\nabla \times \gamma \nabla \times \nabla \times \nabla \times \mathbf{E}, \quad \text { for a.e. } \mathrm{x} \in \mathbb{R}_{-}^{3} .
\end{aligned}
$$

For more details, we cite Ref. [38]. We integrate by parts Eqn. (33) in a way that we reconstruct in the integrand Eqns. (37a) and (37b). Then, we write volume integrals on the left-hand side and surface integrals on the right-hand side, as follows

$$
\begin{gathered}
\int_{\mathbb{R}^{3}}(\nabla \times \nabla \times \mathbf{E}) \cdot \mathbf{\Phi} d \mathbf{r}-\int_{\mathbb{R}_{+}^{3}} k_{0}^{2} \mathbf{E} \cdot \mathbf{\Phi} d \mathbf{r}-\int_{\mathbb{R}_{-}^{3}}\left(k_{0}^{2} \varepsilon \mathbf{E}+k_{0}^{2} \nabla \times \alpha \nabla \times \mathbf{E}+\nabla \times \gamma \nabla \times \nabla \times \nabla \times \mathbf{E}\right) \cdot \mathbf{\Phi} d \mathbf{r} \\
=\int_{\Gamma}\left(\left(\nabla \times \mathbf{E}_{+}-\nabla \times \mathbf{E}_{-}\right) \times \mathbf{n}+k_{0}^{2}\left(\alpha \nabla \times \mathbf{E}_{-}\right) \times \mathbf{n}+\left(\gamma \nabla \times \nabla \times \nabla \times \mathbf{E}_{-}\right) \times \mathbf{n}\right) \cdot \mathbf{\Phi}_{t} d \mathbf{s} .
\end{gathered}
$$


Using Eqns. (37a) and (37b), the left-hand side in (38) vanishes. Since $\boldsymbol{\Phi}$ is arbitrary, we get the second interface condition

$$
\left(\nabla \times \mathbf{E}_{+}-\nabla \times \mathbf{E}_{-}\right) \times \mathbf{n}+k_{0}^{2}\left(\alpha \nabla \times \mathbf{E}_{-}\right) \times \mathbf{n}+\left(\gamma \nabla \times \nabla \times \nabla \times \mathbf{E}_{-}\right) \times \mathbf{n}=0 .
$$

We remark that the number of interface conditions for case 2 is less than the number of the reflected and transmitted fields. More precisely, from the dispersion relation we know that there are two transmitted fields at the interface $\Gamma$ into $\mathbb{R}_{-}^{3}$ and one reflected field into $\mathbb{R}_{+}^{3}$. Hence, there are three unknowns, whereas we got only two equations (interface conditions) to identify them. This system of equations is under-determined and can, therefore, lead to ambiguous solutions. In addition, case 2 does not admit any other weak formulation that may give the necessary number of interface conditions. Thus, the corresponding case is not adequate for describing the propagation of light in Padé MMs. For the same reason, case 8 will be excluded from the investigation of Padé MMs.

\subsubsection{Analysis of Case 3}

The wave-like equation corresponding to case 3 is given by:

$$
\nabla \times \nabla \times \mathbf{E}=k_{0}^{2} \tilde{\varepsilon} \mathbf{E}+k_{0}^{2} \nabla \times \tilde{\alpha} \nabla \times \mathbf{E}+\tilde{\gamma} \nabla \times \nabla \times \nabla \times \nabla \times \mathbf{E} .
$$

Weak formulation: In the generalized sense, for $\mathbf{E}$ verifying the following regularities

$$
\mathbf{E} \in \mathbf{L}_{\text {loc }}^{2}\left(\mathbb{R}^{3}\right), \quad \nabla \times \mathbf{E} \in \mathbf{L}_{\text {loc }}^{2}\left(\mathbb{R}^{3}\right), \quad \text { and } \quad \nabla \times \nabla \times \nabla \times \nabla \times \mathbf{E} \in \mathbf{L}_{\text {loc }}^{2}\left(\mathbb{R}_{-}^{3}\right),
$$

and for $\mathbf{\Phi} \in \mathbf{C}_{0}^{\infty}\left(\mathbb{R}^{3}\right)$, we define the weak formulation corresponding to the wave-like Eqn. (40) as follows

$$
\begin{aligned}
\int_{\mathbb{R}^{3}}(\nabla \times \mathbf{E}) \cdot(\nabla \times \mathbf{\Phi}) d \mathbf{r}=k_{0}^{2} \int_{\mathbb{R}_{+}^{3}} \mathbf{E} \cdot \boldsymbol{\Phi} d \mathbf{r}+k_{0}^{2} \int_{\mathbb{R}_{-}^{3}} \varepsilon \mathbf{E} \cdot \mathbf{\Phi} d \mathbf{r} \\
\quad+k_{0}^{2} \int_{\mathbb{R}_{-}^{3}}(\alpha \nabla \times \mathbf{E}) \cdot(\nabla \times \mathbf{\Phi}) d \mathbf{r}+\int_{\mathbb{R}_{-}^{3}}(\gamma \nabla \times \nabla \times \nabla \times \nabla \times \mathbf{E}) \cdot \mathbf{\Phi} d \mathbf{r}
\end{aligned}
$$

We say that $\mathbf{E}$ is a weak solution to the wave-like Eqn. (40) if it has the regularities (41) and satisfies the weak formulation (42).

Interface conditions: We follow the same principle as in case 2. By means of the equations

$$
\begin{aligned}
& \nabla \times \nabla \times \mathbf{E}=k_{0}^{2} \mathbf{E}, \quad \text { for a.e. } \mathrm{x} \in \mathbb{R}_{+}^{3}, \\
& \nabla \times \nabla \times \mathbf{E}=k_{0}^{2} \varepsilon \mathbf{E}+k_{0}^{2} \nabla \times \alpha \nabla \times \mathbf{E}+\gamma \nabla \times \nabla \times \nabla \times \nabla \times \mathbf{E}, \quad \text { for a.e. } \mathbf{x} \in \mathbb{R}_{-}^{3} .
\end{aligned}
$$

We need to apply just one partial integration on the weak formulation (42) to recover Eqns. (43a) and (43b). Then, we obtain

$$
\begin{array}{r}
\int_{\mathbb{R}_{+}^{3}}(\underbrace{\nabla \times \nabla \times \mathbf{E}-k_{0}^{2} \mathbf{E}}_{=0}) \cdot \mathbf{\Phi} d \mathbf{r}+\int_{\mathbb{R}_{-}^{3}}(\underbrace{\nabla \times \nabla \times \mathbf{E}-k_{0}^{2} \varepsilon \mathbf{E}-k_{0}^{2} \nabla \times \alpha \nabla \times \mathbf{E}-\gamma \nabla \times \nabla \times \nabla \times \nabla \times \mathbf{E}}_{=0}) \cdot \mathbf{\Phi} d \mathbf{r} \\
=\int_{\Gamma}\left(\left(\nabla \times \mathbf{E}_{+}-\nabla \times \mathbf{E}_{-}\right) \times \mathbf{n}+k_{0}^{2}\left(\alpha \nabla \times \mathbf{E}_{-}\right) \times \mathbf{n}\right) \cdot \mathbf{\Phi}_{t} d \mathbf{s}=0 . \quad(44)
\end{array}
$$

For an arbitrary test function $\boldsymbol{\Phi}$, we get the second interface condition

$$
\left(\nabla \times \mathbf{E}_{+}-\nabla \times \mathbf{E}_{-}\right) \times \mathbf{n}+k_{0}^{2}\left(\alpha \nabla \times \mathbf{E}_{-}\right) \times \mathbf{n}=0 .
$$

Because of the same reason as in case 2, which is a lack of additional interface conditions, case 3 will be excluded form the study. This inconsistency is due to the material parameters $\gamma$ that represents the nonlocal effect, which is located in the first position in the fourth order differential operator. Cases 6 and 9 share the same nature of the fourth order term. Hence, case 9 will be immediately excluded in turn. Whereas, because of the nature of the second order term in case 6 , we have a chance to keep it initially in the investigation.

\subsubsection{Analysis of Case 4}

We recall the wave-like equation corresponding to case 4 :

$$
\nabla \times \nabla \times \mathbf{E}=k_{0}^{2} \tilde{\varepsilon} \mathbf{E}+k_{0}^{2} \nabla \times \nabla \times \tilde{\alpha} \mathbf{E}+\nabla \times \nabla \times \tilde{\gamma} \nabla \times \nabla \times \mathbf{E} .
$$


Weak formulation: Eqn. (46) is understood in the generalized sense. For E verifying regularities (27) and for $\boldsymbol{\Phi} \in \mathbf{C}_{0}^{\infty}\left(\mathbb{R}^{3}\right)$, we define the weak formulation corresponding to case 4 as follows

$$
\begin{aligned}
\int_{\mathbb{R}^{3}}(\nabla \times \mathbf{E}) \cdot(\nabla \times \mathbf{\Phi}) d \mathbf{r}=k_{0}^{2} & \int_{\mathbb{R}_{+}^{3}} \mathbf{E} \cdot \mathbf{\Phi} d \mathbf{r}+k_{0}^{2} \int_{\mathbb{R}_{-}^{3}} \varepsilon \mathbf{E} \cdot \mathbf{\Phi} d \mathbf{r} \\
& +k_{0}^{2} \int_{\mathbb{R}_{-}^{3}}(\alpha \mathbf{E}) \cdot(\nabla \times \nabla \times \mathbf{\Phi}) d \mathbf{r}+\int_{\mathbb{R}_{-}^{3}}(\gamma \nabla \times \nabla \times \mathbf{E}) \cdot(\nabla \times \nabla \times \mathbf{\Phi}) d \mathbf{r}
\end{aligned}
$$

We say that $\mathbf{E}$ is a weak solution to the wave-like Eqn. (46) if it has the regularities (27) and satisfies the weak formulation (47).

Interface conditions: We follow the same principle as in cases 2 and 3. By means of the equations

$$
\begin{aligned}
& \nabla \times \nabla \times \mathbf{E}=k_{0}^{2} \mathbf{E}, \quad \text { for a.e. } \mathrm{x} \in \mathbb{R}_{+}^{3}, \\
& \nabla \times \nabla \times \mathbf{E}=k_{0}^{2} \varepsilon \mathbf{E}+k_{0}^{2} \nabla \times \nabla \times \alpha \mathbf{E}+\nabla \times \nabla \times \gamma \nabla \times \nabla \times \mathbf{E}, \quad \text { for a.e. } \mathrm{x} \in \mathbb{R}_{-}^{3},
\end{aligned}
$$

after partial integration, the weak formulation (47) leads to the following formula

$$
\begin{aligned}
\int_{\mathbb{R}_{+}^{3}}(\underbrace{\nabla \times \nabla \times \mathbf{E}-k_{0}^{2} \mathbf{E}}_{=0}) \cdot \mathbf{\Phi} d \mathbf{r}+\int_{\mathbb{R}_{-}^{3}}(\underbrace{\left.\nabla \times \nabla \times \mathbf{E}-k_{0}^{2} \varepsilon \mathbf{E}-k_{0}^{2} \nabla \times \nabla \times \alpha \mathbf{E}-\nabla \times \nabla \times \gamma \nabla \times \nabla \times \mathbf{E}\right) \cdot \mathbf{\Phi} d \mathbf{r}}_{=0} \\
=\int_{\Gamma}\left(\left(\nabla \times \mathbf{E}_{+}-\nabla \times \mathbf{E}_{-}\right) \times \mathbf{n}+k_{0}^{2}\left(\nabla \times \alpha \mathbf{E}_{-}\right) \times \mathbf{n}+\left(\nabla \times \gamma \nabla \times \nabla \times \mathbf{E}_{-}\right) \times \mathbf{n}\right) \cdot \mathbf{\Phi}_{t} d \mathbf{s} \\
\quad+\int_{\Gamma}\left(k_{0}^{2}\left(\alpha \mathbf{E}_{-}\right) \times \mathbf{n}+\left(\gamma \nabla \times \nabla \times \mathbf{E}_{-}\right) \times \mathbf{n}\right) \cdot(\nabla \times \mathbf{\Phi})_{t} d \mathbf{s}=0 . \quad(49)
\end{aligned}
$$

The left-hand side is null because of Eqns. (48a) and (48b). To get out the second interface conditions, we choose the test function $\boldsymbol{\Phi}$ given by

$$
\boldsymbol{\Phi}(\mathbf{r})=\left(\Phi_{1}(x, y) \eta(z), \Phi_{2}(x, y) \eta(z), 0\right)^{T}
$$

where $\Phi_{1}, \Phi_{2}$ are arbitrary functions in $\mathbf{C}_{0}^{\infty}\left(\mathbb{R}^{2}\right)$ and $\eta(z) \in \mathbf{C}_{0}^{\infty}(\mathbb{R})$, satisfying $\left.\eta\right|_{\{z<\mathrm{d}\}}=1$. On the surface $\Gamma$ we have

$$
\begin{aligned}
\left.\boldsymbol{\Phi}\right|_{\Gamma} & =\left(\Phi_{1}, \Phi_{2}, 0\right)^{T} \\
\nabla \times\left.\boldsymbol{\Phi}\right|_{\Gamma} & =\left(0,0, \partial_{x} \Phi_{2}-\partial_{y} \Phi_{1}\right)^{T} .
\end{aligned}
$$

Then, we obtain

$$
\left(\nabla \times \mathbf{E}_{+}-\nabla \times \mathbf{E}_{-}\right) \times \mathbf{n}+k_{0}^{2}\left(\nabla \times \alpha \mathbf{E}_{-}\right) \times \mathbf{n}+\left(\nabla \times \gamma \nabla \times \nabla \times \mathbf{E}_{-}\right) \times \mathbf{n}=0 .
$$

To extract the third interface conditions, we choose the test function $\boldsymbol{\Phi}$ this time in the form

$$
\mathbf{\Phi}(\mathbf{r})=\left(\Phi_{2}(x, y) z \eta(z),-\Phi_{1}(x, y) z \eta(z), 0\right)^{T}
$$

such that $\Phi_{1}, \Phi_{2} \in \mathbf{C}_{0}^{\infty}\left(\mathbb{R}^{2}\right)$ and $\eta(z) \in \mathbf{C}_{0}^{\infty}(\mathbb{R})$, satisfying $\left.\eta\right|_{\{z<\mathrm{d}\}}=1$. On the surface $\Gamma$ we have

$$
\begin{aligned}
\left.\boldsymbol{\Phi}\right|_{\Gamma} & =(0,0,0)^{T} \\
\nabla \times\left.\boldsymbol{\Phi}\right|_{\Gamma} & =\left(\Phi_{1}, \Phi_{2}, 0\right)^{T} .
\end{aligned}
$$

Since $\Phi_{1}$ and $\Phi_{2}$ are arbitrary, we have

$$
k_{0}^{2}\left(\alpha \mathbf{E}_{-}\right) \times \mathbf{n}+\left(\gamma \nabla \times \nabla \times \mathbf{E}_{-}\right) \times \mathbf{n}=0 .
$$

Now, we set all the interface conditions for the case 4 as follows

$$
\begin{aligned}
& \left(\mathbf{E}_{+}-\mathbf{E}_{-}\right) \times \mathbf{n}=0, \\
& \left(\nabla \times \mathbf{E}_{+}-\nabla \times \mathbf{E}_{-}\right) \times \mathbf{n}+k_{0}^{2}\left(\nabla \times \alpha \mathbf{E}_{-}\right) \times \mathbf{n}+\left(\nabla \times \gamma \nabla \times \nabla \times \mathbf{E}_{-}\right) \times \mathbf{n}=0, \\
& k_{0}^{2}\left(\alpha \mathbf{E}_{-}\right) \times \mathbf{n}+\left(\gamma \nabla \times \nabla \times \mathbf{E}_{-}\right) \times \mathbf{n}=0 .
\end{aligned}
$$

The first criterion is satisfied by the case 4 since we have three interface conditions as required. 


\subsubsection{Analysis of Case 5}

The wave equation corresponding to case 5 reads

$$
\nabla \times \nabla \times \mathbf{E}=k_{0}^{2} \tilde{E} \mathbf{E}+k_{0}^{2} \nabla \times \nabla \times \tilde{\alpha} \mathbf{E}+\nabla \times \tilde{\gamma} \nabla \times \nabla \times \nabla \times \mathbf{E} .
$$

Weak formulation: Eqn. (55) is understood in the generalized sense. For $\mathbf{E}$ verifying regularities (32) and for $\boldsymbol{\Phi} \in \mathbf{C}_{0}^{\infty}\left(\mathbb{R}^{3}\right)$, we define the weak formulation corresponding to case 5

$$
\begin{aligned}
\int_{\mathbb{R}^{3}}(\nabla \times \mathbf{E}) \cdot(\nabla \times \mathbf{\Phi}) d \mathbf{r}=k_{0}^{2} & \int_{\mathbb{R}_{+}^{3}} \mathbf{E} \cdot \mathbf{\Phi} d \mathbf{r}+k_{0}^{2} \int_{\mathbb{R}_{-}^{3}} \varepsilon \mathbf{E} \cdot \mathbf{\Phi} d \mathbf{r} \\
& +k_{0}^{2} \int_{\mathbb{R}_{-}^{3}}(\alpha \mathbf{E}) \cdot(\nabla \times \nabla \times \mathbf{\Phi}) d \mathbf{r}+\int_{\mathbb{R}_{-}^{3}}(\gamma \nabla \times \nabla \times \nabla \times \mathbf{E}) \cdot(\nabla \times \mathbf{\Phi}) d \mathbf{r} .
\end{aligned}
$$

We say that $\mathbf{E}$ is a weak solution to the wave-like Eqn. (55) if it has the regularities (32) and satisfies the weak formulation (56).

Interface conditions: We follow the same process as in case 4. By taking the choices (50) and (52) for the test function $\boldsymbol{\Phi}$, we get the following interface conditions

$$
\begin{aligned}
& \left(\mathbf{E}_{+}-\mathbf{E}_{-}\right) \times \mathbf{n}=0, \\
& \left(\nabla \times \mathbf{E}_{+}-\nabla \times \mathbf{E}_{-}\right) \times \mathbf{n}+k_{0}^{2}\left(\nabla \times \alpha \mathbf{E}_{-}\right) \times \mathbf{n}+\left(\gamma \nabla \times \nabla \times \nabla \times \mathbf{E}_{-}\right) \times \mathbf{n}=0, \\
& k_{0}^{2}\left(\alpha \mathbf{E}_{-}\right) \times \mathbf{n}=0 .
\end{aligned}
$$

We obtained three interface conditions as required, then the first criterion is satisfied by the case 5 .

\subsubsection{Analysis of Case 6}

We recall the wave equation corresponding to case 6

$$
\nabla \times \nabla \times \mathbf{E}=k_{0}^{2} \tilde{E} \mathbf{E}+k_{0}^{2} \nabla \times \nabla \times \tilde{\alpha} \mathbf{E}+\tilde{\gamma} \nabla \times \nabla \times \nabla \times \nabla \times \mathbf{E} .
$$

The nature of the second order term in this case gives the possibility to keep it initially in the study, even if the nonlocal material parameter $\tilde{\gamma}$ is located in the first position as in cases 3 and 9 . In such equations, the effect of the nonlocal parameter does not appear explicitly in the interface conditions, but it is present on the level of the dispersion relation.

Weak formulation: In the generalized sense, for $\mathbf{E}$ verifying regularities (41) and for $\boldsymbol{\Phi} \in \mathbf{C}_{0}^{\infty}\left(\mathbb{R}^{3}\right)$, we define the weak formulation corresponding to case 6

$$
\begin{aligned}
\int_{\mathbb{R}^{3}}(\nabla \times \mathbf{E}) \cdot(\nabla \times \mathbf{\Phi}) d \mathbf{r}=k_{0}^{2} \int_{\mathbb{R}_{+}^{3}} \mathbf{E} \cdot \mathbf{\Phi} d \mathbf{r}+k_{0}^{2} \int_{\mathbb{R}_{-}^{3}} \varepsilon \mathbf{E} \cdot \mathbf{\Phi} d \mathbf{r} \\
\quad+k_{0}^{2} \int_{\mathbb{R}_{-}^{3}}(\alpha \mathbf{E}) \cdot(\nabla \times \nabla \times \mathbf{\Phi}) d \mathbf{r}+\int_{\mathbb{R}_{-}^{3}}(\gamma \nabla \times \nabla \times \nabla \times \nabla \times \mathbf{E}) \cdot \mathbf{\Phi} d \mathbf{r} .
\end{aligned}
$$

We say that $\mathbf{E}$ is a weak solution to the wave-like Eqn. (58) if it has the regularities (41) and satisfies the weak formulation (59).

Interface conditions: Analogous to cases 2, 4 and 5, we derive the interface conditions corresponding to case 6 , given by

$$
\begin{aligned}
& \left(\mathbf{E}_{+}-\mathbf{E}_{-}\right) \times \mathbf{n}=0, \\
& \left(\nabla \times \mathbf{E}_{+}-\nabla \times \mathbf{E}_{-}\right) \times \mathbf{n}+k_{0}^{2}\left(\nabla \times \alpha \mathbf{E}_{-}\right) \times \mathbf{n}=0, \\
& k_{0}^{2}\left(\alpha \mathbf{E}_{-} \times \mathbf{n}\right)=0 .
\end{aligned}
$$

\subsubsection{Analysis of Case 7}

We recall the wave equation corresponding to case 7 :

$$
\nabla \times \nabla \times \mathbf{E}=k_{0}^{2} \tilde{E} \mathbf{E}+k_{0}^{2} \tilde{\alpha} \nabla \times \nabla \times \mathbf{E}+\nabla \times \nabla \times \tilde{\gamma} \nabla \times \nabla \times \mathbf{E} .
$$


Weak formulation: Eqn. (61) is understood in the generalized sense. For E verifying regularities (27) and for $\boldsymbol{\Phi} \in \mathbf{C}_{0}^{\infty}\left(\mathbb{R}^{3}\right)$, we define the weak formulation corresponding to case 7

$$
\begin{aligned}
\int_{\mathbb{R}^{3}}(\nabla \times \mathbf{E}) \cdot(\nabla \times \mathbf{\Phi}) d \mathbf{r}=k_{0}^{2} \int_{\mathbb{R}_{+}^{3}} \mathbf{E} \cdot \boldsymbol{\Phi} d \mathbf{r}+k_{0}^{2} \int_{\mathbb{R}_{-}^{3}} \varepsilon \mathbf{E} \cdot \mathbf{\Phi} d \mathbf{r} \\
\quad+k_{0}^{2} \int_{\mathbb{R}_{-}^{3}}(\alpha \nabla \times \nabla \times \mathbf{E}) \cdot \boldsymbol{\Phi} d \mathbf{r}+\int_{\mathbb{R}_{-}^{3}}(\gamma \nabla \times \nabla \times \mathbf{E}) \cdot(\nabla \times \nabla \times \mathbf{\Phi}) d \mathbf{r}
\end{aligned}
$$

We say that $\mathbf{E}$ is a weak solution to the wave-like Eqn. (61) if it satisfies the weak formulation (62) and has the following regularities (27).

Interface conditions: Analogous to cases 2, 4, 5 and 6 , we derive the interface conditions corresponding to case 7 , given by

$$
\begin{aligned}
& \left(\mathbf{E}_{+}-\mathbf{E}_{-}\right) \times \mathbf{n}=0, \\
& \left(\nabla \times \mathbf{E}_{+}-\nabla \times \mathbf{E}_{-}\right) \times \mathbf{n}+\left(\nabla \times \gamma \nabla \times \nabla \times \mathbf{E}_{-}\right) \times \mathbf{n}=0, \\
& \left(\gamma \nabla \times \nabla \times \mathbf{E}_{-}\right) \times \mathbf{n}=0 .
\end{aligned}
$$

The first criterion in the checklist consisted on deriving the right number of interface conditions, which are necessary for computing the amplitudes of all the propagating modes in MMs. The dispersion relation of each case reveals that we need exactly three interface conditions on each surface. Cases 1, 4, 5, 6, and 7 survived, because of fulfilling this criterion. The weak formulation of cases $2,3,8$, and 9 are not adequate to give the additional interface condition. Hence, the systems of equations necessary for defining the amplitudes of all the propagating modes are under-determined, which implies that these cases will be rejected. In the next subsection we study reflection and transmission coefficients for the remaining cases and continue to consider only those that agree in the limiting case of a vanishing nonlocality with expressions obtained from a WSD.

\subsection{Reflection and transmission coefficients analysis}

We recall that the main motivation for proceeding with the nonlocal analysis is to overcome the limitations exhibited when using the local theory. This fact implies that the local approach must be contained in the nonlocal approach. Meaning, by taking the limit of the parameters representing the nonlocality to zero, we have to recover the local models. On the level of the mathematical models this can be clearly seen from equations in Tab. 1. However, for more accurate analysis we need to check the limit of the corresponding interface conditions and also the produced reflected and transmitted modes. Already, by using the local approach, we know that we have only one reflected and one transmitted mode in each half-space. Besides, for the nonlocal models we have one reflected mode together with two transmitted modes. This fact implies that by taking the limit of the material parameter $\gamma$ to zero, one of that two transmitted modes must vanish. Actually, we can also observe that on the level of the interface conditions. In other words, one of the three interface conditions vanish when $\gamma$ tends to zero. For this reason, we present a quick check for the remaining cases 1, 4, 5, 6, and 7. Then, we need to recall the principle of Fresnel equations that serve to compute the amplitudes of the reflected and transmitted modes, and we give their formulae for the surviving cases.

\subsubsection{Analysis of the remaining cases using the second criterion}

Analysis of Case 1: We take the limit of $\gamma$ to zero in the interface conditions corresponding to case 1 , we get

$$
\begin{aligned}
& \left(\mathbf{E}_{+}-\mathbf{E}_{-}\right) \times \mathbf{n}=0, \\
& \left(\nabla \times \mathbf{E}_{+}-\nabla \times \mathbf{E}_{-}\right) \times \mathbf{n}+k_{0}^{2}\left(\alpha \nabla \times \mathbf{E}_{-}\right) \times \mathbf{n}=0 .
\end{aligned}
$$

We notice that using the notation $\mu=\left(1-k_{0}^{2} \alpha\right)^{-1}$ (cf. Subsec. 4.2), the limit interface conditions take the same formulae as in presence of WSD, as follows

$$
\begin{aligned}
& \left(\mathbf{E}_{+}-\mathbf{E}_{-}\right) \times \mathbf{n}=0 \\
& \left(\nabla \times \mathbf{E}_{+}-\mu^{-1} \nabla \times \mathbf{E}_{-}\right) \times \mathbf{n}=0
\end{aligned}
$$

Which implies that the second criterion is fulfilled by the first case. 
Analysis of Case 4: In presence of WSD, the wave-like Eqn. (46) reads

$$
\nabla \times \nabla \times \mathbf{E}=k_{0}^{2} \tilde{\varepsilon} \mathbf{E}+k_{0}^{2} \nabla \times \nabla \times \tilde{\alpha} \mathbf{E} .
$$

Its corresponding interface conditions are given by

$$
\begin{aligned}
& \left(\mathbf{E}_{+}-\mathbf{E}_{-}\right) \times \mathbf{n}=0, \\
& \left(\nabla \times \mathbf{E}_{+}-\nabla \times \mathbf{E}_{-}\right) \times \mathbf{n}+k_{0}^{2}\left(\nabla \times \alpha \mathbf{E}_{-}\right) \times \mathbf{n}=0 .
\end{aligned}
$$

On the other hand, the limiting interface conditions for case 4 , when $\gamma$ tends to zero read

$$
\begin{aligned}
& \left(\mathbf{E}_{+}-\mathbf{E}_{-}\right) \times \mathbf{n}=0, \\
& \left(\nabla \times \mathbf{E}_{+}-\nabla \times \mathbf{E}_{-}\right) \times \mathbf{n}+k_{0}^{2}\left(\nabla \times \alpha \mathbf{E}_{-}\right) \times \mathbf{n}=0, \\
& k_{0}^{2}\left(\alpha \mathbf{E}_{-}\right) \times \mathbf{n}=0 .
\end{aligned}
$$

We can clearly see that the presence of the material parameter $\alpha$ in the third interface condition prevents it to vanish. Meanwhile, the dispersion relation for $\gamma \rightarrow 0$ states that there is only one transmitted field in the halfspace filled with the MM. In this case, the system of equations is overdetermined and leads to unphysical results. From a physical point of view, the third interface condition states that the tangential component of $\mathbf{E}_{-}$is zero at the interface. Linking this with the first (natural) interface condition, would suggest that the tangential component of $\mathbf{E}_{+}$, i.e., in vacuum is zero. Since the total field in the incidence half-space is $\mathbf{E}^{I}+\mathbf{E}^{R}$, the transversal components of the incident and reflected fields totally cancel, independent of the angle of incidence, the permittivity contrast between the two half-spaces and polarization. The result is even stranger when the MM is lossy ,i.e., when $\Im \varepsilon>0$, where some energy is absorbed in the MM half-space. However, here, we obtain a total reflection phenomenon, without incorporating loss. This is clearly a contradiction to the classical reflection and transmission phenomena between two local materials.

This case represents a counterexample that shows that even if we have the exact number of interface conditions in presence of SSD, that gives the exact number of reflected and transmitted modes in turn, it is not possible to reproduce the local forward and backward modes after taking the limit of the nonlocal parameter to zero. To conclude, the case 4 does not fulfill the second criterion, and will, therefore, be excluded.

For the same reason of no conformity with the modes produced by the WSD when taking the limit of $\gamma$ to zero, cases 5 and 6 will be excluded from the investigation of Padé MMs.

Analysis of Case 7: When we take the limit of $\gamma$ to zero, i.e., in presence of WSD, the wave-like Eqn. (61) reads

$$
\nabla \times \nabla \times \mathbf{E}=k_{0}^{2} \tilde{\varepsilon} \mathbf{E}+k_{0}^{2} \tilde{\alpha} \nabla \times \nabla \times \mathbf{E} .
$$

Its corresponding interface conditions are given by

$$
\begin{aligned}
& \left(\mathbf{E}_{+}-\mathbf{E}_{-}\right) \times \mathbf{n}=0, \\
& \left(\nabla \times \mathbf{E}_{+}-\nabla \times \mathbf{E}_{-}\right) \times \mathbf{n}=0 .
\end{aligned}
$$

They represent, exactly, the same limiting interface conditions for case 7 when $\gamma$ tends to zero. Which implies that the second criterion is fulfilled in this case.

\subsubsection{Fresnel coefficients for Cases 1 and 7}

We denote the incident, reflected and transmitted fields by the superscripts I, R, and T, respectively, and we refer to each one of them by $*$. Inside the slab MM, we have four propagating linearly independent eigenmodes. Two modes are forward and the other two ones are backward, we refer to each one by $\mathbf{E}^{j}$, for $j \in\{1,2,3,4\}$. The total field in the incidence half-space is given by $\mathbf{E}^{I}+\mathbf{E}^{R}$. Inside the MM slab, the total field is the sum of the propagating eigenmodes. It is written $\mathbf{E}^{\text {slab }}=\sum_{j=1}^{4} \mathbf{E}^{j}$. In the transmission plane, we have just one transmitted field $\mathbf{E}^{T}$ (see Fig. 2).

The reflection $(\rho)$ and transmission $(\tau)$ coefficients represent the ratios of the amplitudes of the reflected and transmitted waves to the incident wave. We compute the amplitudes of these fields by using Fresnel formulae. We start by plugging the plane wave ansatzes into the interface conditions defined on the surfaces $\Gamma_{+}$and $\Gamma_{-}$. For both polarizations, we have

- TE-polarization:

$$
\begin{aligned}
& \mathbf{E}^{*}=\mathbf{E}_{0}^{*} \exp \left(\mathrm{ik}^{*} \cdot \mathbf{r}\right), \text { with } \quad \mathbf{E}_{0}^{*}=\left(E_{x}^{*}, 0,0\right)^{T}, \text { and } \quad \mathbf{k}^{*}=\left(0, k_{y}, k_{z}^{*}\right)^{T} . \\
& \mathbf{E}^{j}=\mathbf{E}_{0}^{j} \exp \left(\mathrm{ik}^{*} \cdot \mathbf{r}\right), \text { with } \quad \mathbf{E}_{0}^{j}=\left(E_{x}^{j}, 0,0\right)^{T}, \text { and } \quad \mathbf{k}^{j}=\left(0, k_{y}, k_{z}^{j}\right)^{T} .
\end{aligned}
$$




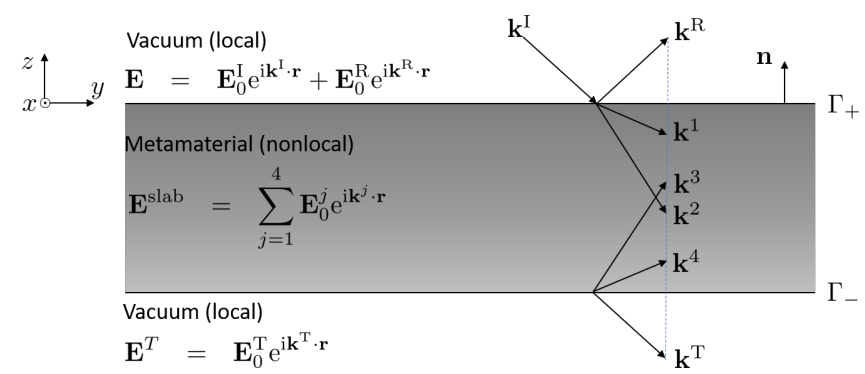

Figure 2: Propagating modes inside a slab MM. We have two forward modes defined on the the surfce $\Gamma^{+}$and the other backward modes are defined on the surface $\Gamma^{-}$.

- TM-polarization:

$$
\begin{aligned}
& \mathbf{E}^{*}=\mathbf{E}_{0}^{*} \exp \left(\mathrm{ik} \mathbf{k}^{*} \cdot \mathbf{r}\right), \text { with } \quad \mathbf{E}_{0}^{*}=\left(0, E_{y}^{*}, E_{z}^{*}\right)^{T}, \text { and } \quad \mathbf{k}^{*}=\left(0, k_{y}, k_{z}^{*}\right)^{T} . \\
& \mathbf{E}^{j}=\mathbf{E}_{0}^{j} \exp \left(\mathrm{ik}^{*} \cdot \mathbf{r}\right), \text { with } \quad \mathbf{E}_{0}^{j}=\left(0, E_{y}^{j}, E_{z}^{j}\right)^{T}, \text { and } \quad \mathbf{k}^{j}=\left(0, k_{y}, k_{z}^{j}\right)^{T} .
\end{aligned}
$$

Using the divergence free equation for the electric displacement field $\mathbf{D}$, we get a relation between the $y$ and $z$ components of the electric field $\mathbf{E}$, that depends on the medium and is given by

$$
\left\{\begin{array}{l}
E_{y}^{*}=-\frac{k_{z}^{*}}{k_{y}} E_{z}^{*}, \quad \text { in } \Omega_{-} \cup \Omega_{+} . \\
E_{y}^{j}=-\frac{\varepsilon_{z} k_{z}^{*}}{\varepsilon_{y} k_{y}} E_{z}^{j}, \quad \text { in } \Omega_{\delta} .
\end{array}\right.
$$

Then, we get an algebraic system in the form

$$
\mathcal{A} E=\mathcal{F} .
$$

In total we have six unknowns for each polarization, such that $E=\left(E_{(P)}^{R}, E_{(P)}^{1}, E_{(P)}^{2}, E_{(P)}^{3}, E_{(P)}^{4}, E_{(P)}^{T}\right)^{T}$. The index $(P)$ refers to the $x$ component of the electric field for TE polarization and to the $z$ component of the electric field for TM polarization. The Fresnel matrix $\mathcal{A}$ is a $6 \times 6$ matrix, obtained from the fact that we have on each surface of the slab three interface conditions leading to three equations. The non-homogeneity is described by the vector $\mathcal{F}$, which is relative to the incident plane wave.

In the sequel, we give Fresnel matrices for TE and TM polarizations separately. Each matrix combines Fresnel coefficients for the cases 1 and 7 , followed with tables precising the explicit formulae of some coefficients. Fresnel formulae of the case 1 are computed and written clearly in [41].

\section{Fresnel matrix for TE-polarization}

$$
\mathcal{A}^{(T E)}=\left(\begin{array}{cccccc}
1 & -1 & -1 & -1 & -1 & 0 \\
k_{z}^{R} & b_{1}^{(T E)} & b_{2}^{(T E)} & b_{3}^{(T E)} & b_{4}^{(T E)} & 0 \\
0 & c_{1}^{(T E)} & c_{2}^{(T E)} & c_{3}^{(T E)} & c_{4}^{(T E)} & 0 \\
0 & -\mathrm{e}^{\mathrm{i} k_{z}^{1} d} & -\mathrm{e}^{\mathrm{i} k_{z}^{2} d} & -\mathrm{e}^{\mathrm{i} k_{z}^{3} d} & -\mathrm{e}^{\mathrm{i} k_{z}^{4} d} & 1 \\
0 & c_{1}^{(T E)} \mathrm{e}^{\mathrm{i} k_{z}^{1} d} & c_{2}^{(T E)} \mathrm{e}^{\mathrm{i} k_{z}^{2} d} & c_{3}^{(T E)} \mathrm{e}^{\mathrm{i} k_{z}^{3} d} & c_{4}^{(T E)} \mathrm{e}^{\mathrm{i} k_{z}^{4} d} & 0 \\
0 & b_{1}^{(T E)} \mathrm{e}^{\mathrm{i} k_{z}^{1} d} & b_{2}^{(T E)} \mathrm{e}^{\mathrm{i} k_{z}^{2} d} & b_{3}^{(T E)} \mathrm{e}^{\mathrm{i} k_{z}^{3} d} & b_{4}^{(T E)} \mathrm{e}^{\mathrm{i} k_{z}^{4} d} & k_{z}^{T}
\end{array}\right),
$$

and $\mathcal{F}^{(T E)}=\left(-1,-k_{z}^{I}, 0,0,0,0\right)^{T}$. The coefficients $b_{j}^{(T E)}$ and $c_{j}^{(T E)}$ for both cases 1 and 7 and $1 \leq j \leq 4$ are given in Tab. 3.

\begin{tabular}{ccc}
\hline & Case 1 & Case 7 \\
\hline$b_{j}^{(T E)}$ & $-\left(1-k_{0}^{2} \alpha_{y}-\gamma_{x}\left(\mathbf{k}^{j}\right)^{2}\right) k_{z}^{j}$ & $-\left(1-\gamma_{x}\left(\mathbf{k}^{j}\right)^{2}\right) k_{z}^{j}$ \\
$c_{j}^{(T E)}$ & $\gamma_{x}\left(\mathbf{k}^{j}\right)^{2}$ & $\gamma_{x}\left(\mathbf{k}^{j}\right)^{2}$ \\
\hline
\end{tabular}

Table 3: Substitution of the coefficients $b_{j}^{(T E)}$ and $c_{j}^{(T E)}$, for $1 \leq j \leq 4$, corresponding to TE polarization for the cases 1 and 7 ; with $\left(\mathbf{k}^{j}\right)^{2}=\left(k_{y}\right)^{2}+\left(k_{z}^{j}\right)^{2}$. 


\section{Fresnel matrix for TM-polarization}

$$
\mathcal{A}^{(T M)}=\left(\begin{array}{cccccc}
k_{z}^{R} & -\frac{\varepsilon_{z}}{\varepsilon_{y}} k_{z}^{1} & -\frac{\varepsilon_{z}}{\varepsilon_{y}} k_{z}^{2} & -\frac{\varepsilon_{z}}{\varepsilon_{y}} k_{z}^{3} & -\frac{\varepsilon_{z}}{\varepsilon_{y}} k_{z}^{4} & 0 \\
\left(\mathbf{k}^{R}\right)^{2} & b_{1}^{(T M)} & b_{2}^{(T M)} & b_{3}^{(T M)} & b_{4}^{(T M)} & 0 \\
0 & c_{1}^{(T M)} & c_{2}^{(T M)} & c_{3}^{(T M)} & c_{4}^{(T M)} & 0 \\
0 & -\frac{\varepsilon_{z}}{\varepsilon_{y}} k_{z}^{1} \mathrm{e}^{\mathrm{i} k_{z}^{1} d} & -\frac{\varepsilon_{z}}{\varepsilon_{y}} k_{z}^{2} \mathrm{e}^{\mathrm{i} k_{z}^{2} d} & -\frac{\varepsilon_{z}}{\varepsilon_{y}} k_{z}^{3} \mathrm{e}^{\mathrm{i} k_{z}^{3} d} & -\frac{\varepsilon_{z}}{\varepsilon_{y}} k_{z}^{4} \mathrm{e}^{\mathrm{i} k_{z}^{4} d} & 1 \\
0 & c_{1}^{(T M)} \mathrm{e}^{\mathrm{i} k_{z}^{1} d} & c_{2}^{(T M)} \mathrm{e}^{\mathrm{i} k_{z}^{2} d} & c_{3}^{(T M)} \mathrm{e}^{\mathrm{i} k_{z}^{3} d} & c_{4}^{(T M)} \mathrm{e}^{\mathrm{i} k_{z}^{4} d} & 0 \\
0 & b_{1}^{(T M)} \mathrm{e}^{\mathrm{i} k_{z}^{1} d} & b_{2}^{(T M)} \mathrm{e}^{\mathrm{i} k_{z}^{2} d} & b_{3}^{(T M)} \mathrm{e}^{\mathrm{i} k_{z}^{3} d} & b_{4}^{(T M)} \mathrm{e}^{\mathrm{i} k_{z}^{4} d} & \left(\mathbf{k}^{T}\right)^{2}
\end{array}\right),
$$

and $\mathcal{F}^{(T M)}=\left(-1,-\left(\mathbf{k}^{I}\right)^{2}, 0,0,0,0\right)^{T}$. The coefficients $b_{j}^{(T M)}$ and $c_{j}^{(T M)}$ for both cases 1 and 7 and $1 \leq j \leq 4$ are given in Tab. 4

\begin{tabular}{ccc}
\hline & Case 1 & Case 7 \\
\hline$b_{j}^{(T M)}$ & $-\left[1-\gamma_{z}\left(k_{y}\right)^{2}-\gamma_{y}\left(k_{z}^{j}\right)^{2}\right]\left[\left(k_{y}\right)^{2}+\frac{\varepsilon_{z}}{\varepsilon_{y}}\left(k_{z}^{j}\right)^{2}\right]$ & $-\left[1-\gamma_{z}\left(k_{y}\right)^{2}-\gamma_{y}\left(k_{z}^{j}\right)^{2}\right]\left[\left(k_{y}\right)^{2}+\frac{\varepsilon_{z}}{\varepsilon_{y}}\left(k_{z}^{j}\right)^{2}\right]$ \\
$c_{j}^{(T M)}$ & $\gamma_{y}\left[\left(k_{y}\right)^{2}+\frac{\varepsilon_{z}}{\varepsilon_{y}}\left(k_{z}^{j}\right)^{2}\right] k_{z}^{j}$ & $\gamma_{y}\left[\left(k_{y}\right)^{2}+\frac{\varepsilon_{z}}{\varepsilon_{y}}\left(k_{z}^{j}\right)^{2}\right] k_{z}^{j}$ \\
\hline
\end{tabular}

Table 4: Substitution of the coefficients $b_{j}^{(T M)}$ and $c_{j}^{(T M)}$, for $1 \leq j \leq 4$, corresponding to TM polarization for the cases 1 and 7 .

In both polarizations, the notation $\left(\mathbf{k}^{*}\right)^{2}$ means the sum $\left(k_{y}\right)^{2}+\left(k_{z}^{*}\right)^{2}$.

For both cases 1 and 7, taking the limit of the nonlocal parameter $\gamma$ towards zero, we get Fresnel matrices identical to those obtained by considering local constitutive relations. At the level of reflection and transmission coefficients, everything seems to be fine with the cases 1 and 7 . Then, we have to move to the third and final criterion to end up the checking process.

To summarize this section, the second criterion consists on analyzing the reflection and transmission coefficients. As the models produced by the WSD are implicitly included in those obtained in presence of SSD, then we checked whether it is really the case for the remaining proposed cases. Cases 1 and 7 survived, because we could reproduce the same equations as in presence of WSD. Contrary to cases 4,5 , and 6 , their weak formulations led to well posed systems; but the produced interface condition are not valid for computing the right reflection and transmission coefficients corresponding to the WSD.

\subsection{Casimir-Onsager Reciprocity}

The last condition in our checklist is the requirement of time-reversal symmetry for reciprocal media. In fact, it holds that the displacement field $\tilde{\mathbf{D}}(t, \mathbf{r})$ and the electric field $\tilde{\mathbf{E}}(t, \mathbf{r})$ are symmetric under time inversion, but antisymmetric under space inversion (parity); and the magnetic flux $\tilde{\mathbf{B}}(t, \mathbf{r})$ and the magnetic field $\tilde{\mathbf{H}}(t, \mathbf{r})$ are antisymmetric under time inversion, but symmetric under space inversion [57]. These symmetries of the fields have consequences on the symmetry of the nonlocal response function in Fourier space. Due to this fact and to Eqn. (8), we deduce that the response function $\mathbf{R}(\omega, \mathbf{r})$ must contain some symmetry as well. In the spatial frequency space it holds [60]

$$
\widehat{R}_{i j}(\omega, \mathbf{k})=\widehat{R}_{j i}(\omega, \mathbf{k})
$$

which represents the Casimir-Onsager reciprocity principle for centrosymmetric structures. In the remaining case 7, we argue with Casimir-Onsager reciprocity that this case can only exist if $\alpha$ is a scalar (or a multiple of the identity matrix). In that case, there is no need to distinguish between cases 1 and 7 , i.e., case 7 turns out to be a special case of case 1 with the restriction of $\alpha_{i i}=\alpha_{j j}$, for $(i, j) \in\{x, y, z\} \times\{x, y, z\}$. For that matter, let's first look at the term proportional to $\alpha$, i.e. $\gamma=0$. The general expression of the nonlocal response function $\mathbf{R}$ is

$$
D_{i}(\omega, \mathbf{r})=R_{i j}(\omega, \mathrm{i} \nabla) E_{j}(\omega, \mathbf{r})=\varepsilon_{i j} E_{j}(\omega, \mathbf{r})+c_{i j l m} \partial_{l} \partial_{m} E_{j}(\omega, \mathbf{r}) .
$$

According to symmetry condition (69), it must hold that

$$
c_{i j l m}=c_{j i l m} .
$$

We further require that the electric field $\mathbf{E}$ is at least a $\mathcal{C}^{2}\left(\mathbb{R}_{-}^{3}\right)$-function. Consequently, according to the equality of mixed partials (Schwarz's theorem), the second-order derivatives can be interchanged which renders

$$
c_{i j l m} \partial_{l} \partial_{m} E_{j}=c_{i j l m} \partial_{m} \partial_{l} E_{j}=c_{i j m l} \partial_{l} \partial_{m} E_{j} .
$$


In the first equality we put the fact that $\mathbf{E} \in \mathcal{C}^{2}\left(\mathbb{R}_{-}^{3}\right)$ and in the second equation, we simply relabeled the indices. In fact, we have

$$
c_{i j l m}=c_{j i l m}=c_{i j m l}=c_{j i m l} .
$$

These are the fundamental symmetry conditions for the fourth-rank tensor $c_{i j l m}$.

\subsubsection{Study of Case 1 with $\tilde{\gamma}=0$}

Let $\mathbf{D}(\omega, \mathbf{r})=\tilde{\varepsilon} \mathbf{E}(\omega, \mathbf{r})+\nabla \times \tilde{\alpha} \nabla \times \mathbf{E}(\omega, \mathbf{r})$. Inside the homogenized MM, this constitutive relation requires that

$$
c_{i j l m} \partial_{l} \partial_{m} E_{j} \stackrel{!}{=}[\nabla \times(\alpha \nabla \times \mathbf{E})]_{i} .
$$

We develop the right hand side in (73), we get

$$
\nabla \times(\alpha \nabla \times \mathbf{E})=\left(\begin{array}{l}
\alpha_{y y} \partial_{x} \partial_{z} E_{z}-\alpha_{y y} \partial_{z} \partial_{z} E_{x}+\alpha_{z z} \partial_{x} \partial_{y} E_{y}-\alpha_{z z} \partial_{y} \partial_{y} E_{x} \\
\alpha_{x x} \partial_{y} \partial_{z} E_{z}-\alpha_{x x} \partial_{z} \partial_{z} E_{y}+\alpha_{z z} \partial_{x} \partial_{y} E_{x}-\alpha_{z z} \partial_{x} \partial_{x} E_{y} \\
\alpha_{x x} \partial_{y} \partial_{z} E_{x}-\alpha_{x x} \partial_{y} \partial_{y} E_{z}+\alpha_{y y} \partial_{x} \partial_{z} E_{x}-\alpha_{y y} \partial_{x} \partial_{x} E_{z}
\end{array}\right)
$$

where it has been assumed that $\alpha$ is a diagonal matrix. Comparing the coefficients yields

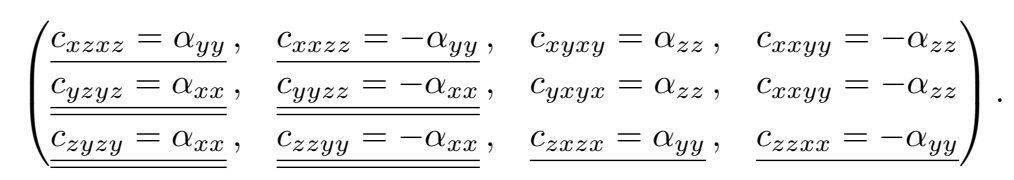

The coefficients in the same number of underlines are related to the same material parameter $\alpha_{i i}$. From the comparison, we note that the assumption in Eqn. (73) is compatible with the fundamental symmetry constraints in Eqn. (72). For example we systematically obtain equalities such as $c_{x y x y}=c_{y x y x}=\alpha_{z z}$ or $c_{y z y z}=c_{z y z y}=\alpha_{x x}$.

We also note that there are constraints that impose $c_{i i j j}=c_{j j i i}$ for all $(i, j) \in\{x, y, z\} \times\{x, y, z\}$ and $i \neq j$, which are not part of the fundamental symmetry constraints. For instance, we have $c_{x x z z}=c_{z z x x}=-\alpha_{y y}$. Furthermore the fact that such terms differ by a minus sign as well, i.e., $c_{i j i j}=-c_{i i j j}$ for all $(i, j) \in\{x, y, z\} \times$ $\{x, y, z\}$ and $i \neq j$ suggests that assumption in Eqn. (73) is of higher symmetry than simply spatial inversion symmetry. The first constraints, i.e., $c_{i i j j}=c_{j j i i}$ for all $(i, j) \in\{x, y, z\} \times\{x, y, z\}$ and $i \neq j$ renders the crystal of a tetragonal system, i.e., of fourfold symmetry. They define the symmetry classes $C_{4}$ and $D_{4 h}$. We stress that the fishnet $\mathrm{MM}$ is of $D_{2 h}$ symmetry (only), while we assume Eqn. (73) to hold. We technically try to describe a system with lower symmetry $\left(D_{2 h}\right)$ with coefficients of higher symmetry $\left(D_{4 h}\right)$. We highlight that the second constraint $c_{i j i j}=-c_{i i j j}$ for all $(i, j) \in\{x, y, z\} \times\{x, y, z\}$ and $i \neq j$, is not linked to a symmetry.

One can prove that the symmetry condition is satisfied by the fourth order term, i.e., when $\tilde{\gamma} \neq 0$. We Follow the same principles as in the second order term, i.e., symmetry condition (69) and the mixed partial derivatives, we can examine the assumption

$$
e_{i j k l m n} \nabla_{k} \nabla_{l} \nabla_{m} \nabla_{n} E_{j} \stackrel{!}{=}[\nabla \times \nabla \times(\gamma(\omega) \nabla \times \nabla \times \mathbf{E})]_{i}
$$

Due to having long formulae, we do not present the computational details in this paper.

\subsubsection{Study of Case 7 with $\tilde{\gamma}=0$}

Let $\mathbf{D}(\omega, \mathbf{r})=\tilde{\epsilon} \mathbf{E}(\omega, \mathbf{r})+\tilde{\alpha} \nabla \times \nabla \times \mathbf{E}(\omega, \mathbf{r})$

Here, $\tilde{\alpha}$ is positioned on the left. This supposition imposes that

$$
c_{i j l m} \partial_{l} \partial_{m} E_{j} \stackrel{!}{=}(\alpha \nabla \times \nabla \times \mathbf{E})_{i} .
$$

We develop the right hand side in (76), we get

$$
\alpha \nabla \times \nabla \times \mathbf{E}=\left(\begin{array}{l}
\alpha_{x x} \partial_{x} \partial_{y} E_{y}-\alpha_{x x} \partial_{y} \partial_{y} E_{x}+\alpha_{x x} \partial_{x} \partial_{z} E_{z}-\alpha_{x x} \partial_{z} \partial_{z} E_{x} \\
\alpha_{y y} \partial_{x} \partial_{y} E_{x}-\alpha_{y y} \partial_{x} \partial_{x} E_{y}+\alpha_{y y} \partial_{y} \partial_{z} E_{z}-\alpha_{y y} \partial_{z} \partial_{z} E_{y} \\
\alpha_{z z} \partial_{x} \partial_{z} E_{x}-\alpha_{z z} \partial_{x} \partial_{x} E_{z}+\alpha_{z z} \partial_{y} \partial_{z} E_{y}-\alpha_{z z} \partial_{y} \partial_{y} E_{z}
\end{array}\right)
$$

Comparing the coefficients yields

$$
\left(\begin{array}{lllll}
\frac{c_{x y x y}=\alpha_{x x}}{\overline{c_{y x y x}=\alpha_{y y}}}, & \frac{c_{x x y y}=-\alpha_{x x}}{\overline{a_{y y x x}=-\alpha_{y y}}}, & \frac{\underline{c_{x z x z}=\alpha_{x x}}}{c_{z x z y z}=\alpha_{y y}}, & \frac{\underline{c_{x x z z}=-\alpha_{x x}}}{\overline{c_{y y z z}=-\alpha_{y y}}}
\end{array}\right) .
$$


From this comparison, we read out that the assumption in Eqn. (76) yields that $c_{x y x y}=\alpha_{x x}, c_{y x y x}=\alpha_{y y}$ and $c_{x z x z}=\alpha_{x x}, c_{z y z y}=\alpha_{z z}$ hold simultaneously. Following the fundamental symmetry constraints (72), it must hold that

$$
\alpha_{x x}=\alpha_{y y}=\alpha_{z z}
$$

Consequently, the system has to be isotropic, otherwise physical symmetries are violated. In this case, the position of $\tilde{\alpha}$ among the curl operators is irrelevant and can be placed in another position. Hence, this case is essentially trivial and can be considered as a special case of model 1 .

As a concluding remark, using Casimir-Onsager reciprocity as a third criterion, we end up with a further elimination of case 7 , as it can only exist if $\alpha$ is a scalar and, therefore, is a special case of model 1.

\section{Conclusion}

In this contribution, we investigated the propagation of light in mediums constituted of MMs, where the unit cells are centro-symmetric and we considered nonlocal constitutive relations. By means of a second-order Padé-type approximation of the response functions, we modeled the light-matter interaction with nine different constitutive relations that lead to different wave equations. The main reason of having this diversity is the nature of the material parameters, which are supposed to be anisotropic diagonal matrices. That means, they do not commute with the differential operators, then, they do not express necessarily the same light-matter interaction. Hence, a checklist process was of major necessity to decide which formulation is suitable for describing the considered optical phenomena. This analysis is mandatory prior checking the validity of a model to adequately homogenize a MM. A valid model must simultaneously fulfill the following three criteria:

- The first criterion concerns the analysis of interface conditions posed on surfaces separating MMs from vacuum. They are obtained by means of the weak formulations corresponding to each case and they are necessary for computing the reflected and transmitted fields. These propagating modes represent the solutions to the dispersion relation relative to each case with respect to TE and TM polarizations. To compute the amplitudes of the reflected and transmitted fields, we derive the corresponding Fresnel formulae. For well defined systems, the number of propagating modes within MMs must coincide with the number of the derived interface conditions. Cases 1, 4, 5, 6, and 7 led to weak formulations that revealed the required number of interface conditions. For cases 2, 3, 8, and 9 it was not the case; the associated systems of equations obtained by using Fresnel formulae are under-determined due to the lack of additional interface conditions. Thus, they are rejected from the investigation for not fulfilling the first criterion.

- The second criterion is called the reflection and transmission coefficients analysis. In principle, by taking the limit of the nonlocal material parameters to zero, we require to reproduce the reflection and transmission coefficients produced by the WSD, as the constitutive relations reduce to those of the local models. More precisely, by following the local approach we get only two propagating fields at each interface. One of them is reflected and the other one is transmitted. In the nonlocal analysis approach, we have three fields propagating away from an interface, one reflected and two transmitted. By taking the limit of the nonlocal material parameter to zero, one of the two transmitted fields must vanish, which is not the case for cases 4, 5, and 6. Namely, for these three cases, if the second transmitted mode vanishes, it implies one of two facts: (a) either the material parameter related to the second order terms in the wave-like equations is zero, which does not even reflect models with WSD, (b) or the tangential components of the transmitted electric field are zero. The latter result would imply a total reflection, that is independent from the angle of incidence and the permittivies of the two materials. Both significations are not true. This fact undeniably rises from the nonvanishing additional interface condition, which makes the system of equations being too restrictive and overdetermined. For cases 1 and 7 this criterion is fulfilled.

- The third and last criterion was based on the requirement of fulfilling the Casimir-Onsager reciprocity. It is a symmetry constraint, which is satisfied by the first case, whereas, for the remaining case 7, it makes sense only when the material parameter related to the second order term is a scalar. It means that case 7 is a special case of the first case.

At the end of the checklist, the only surviving case is the first one, in which the material parameters are sandwiched between an equal number of curl operators. It represents the model studied previously [30] by means of a Taylor approximation of the response function. It showed a good description of the propagation of light in MMs compared to the WSD. The use of Padé-type approximation for the response function opened the path to derive several representation of the light-matter interaction. This approach couldn't improve the quality of the model proposed in [30], but it allowed us to set a solid background to study and check the validity of other models, not necessarily obtained by following the same approach. For further investigations, the Padé-type 
approximation confirms that going to higher order spacial dispersion is required. However, other constitutive relations could have been suggested as well but prior being considered in detail, they have to necessarily pass the checklist that we have put forward in this contribution. Therefore, our work is quite general and important in the ongoing endeavor to homogenize MMs with advanced and nonlocal constitutive relations.

\section{Acknowledgements}

The authors gratefully acknowledge financial support by the Deutsche Forschungsgemeinschaft (DFG) through - Project-ID 258734477 - SFB 1173 and - Project-ID 390761711 - EXC 2082/1. K.M. acknowledges support from the Karlsruhe School of Optics and Photonics (KSOP). A.K. also acknowledges support from the Austrian Science Fund (FWF) under Project No. M 2310-N32.

\section{Appendix: Exact coefficients of the dispersion relations}

In this appendix, we shall summarize the resulting coefficients that appear in every case. While the physical discussion is made in Sec. 5, here we merely write down the exact solutions. For each case, the solution and its corresponding coefficients are written first in TM and then in TE polarization.

Case $1[\hat{\mathbf{p}}(\omega, \mathbf{k})=-\mathbf{k} \times \alpha \mathbf{k} \times$ and $\hat{\mathbf{q}}(\omega, \mathbf{k})=-\mathbf{k} \times \mathbf{k} \times \gamma]$

TM polarization:

$$
k_{z}^{2}\left(k_{y}\right)=-\frac{1}{2}\left(q_{0}^{\mathrm{TM}}+q_{1}^{\mathrm{TM}}\right) k_{y}^{2}+p_{0}^{\mathrm{TM}} \pm \sqrt{l_{0}^{\mathrm{TM}}+\left(p_{0}^{\mathrm{TM}}+\frac{q_{0}^{\mathrm{TM}}-q_{1}^{\mathrm{TM}}}{2} k_{y}^{2}\right)^{2}}
$$

with

$$
q_{0}^{\mathrm{TM}}=\frac{\epsilon_{y}}{\epsilon_{z}}, \quad q_{1}^{\mathrm{TM}}=\frac{\gamma_{z}}{\gamma_{y}}, \quad p_{0}^{\mathrm{TM}}=\frac{-1}{2 \gamma_{y} \mu_{x}}, \quad l_{0}^{\mathrm{TM}}=\frac{k_{0}^{2} \epsilon_{y}}{\gamma_{y}} .
$$

TE polarization:

$$
k_{z}^{2}\left(k_{y}\right)=-k_{y}^{2}+p_{0}^{\mathrm{TE}} \pm \sqrt{l_{0}^{\mathrm{TE}}+\left(p_{0}^{\mathrm{TE}}\right)^{2}+2\left(p_{1}^{\mathrm{TE}}-p_{0}^{\mathrm{TE}}\right) k_{y}^{2}}
$$

with

$$
p_{0}^{\mathrm{TE}}=\frac{-1}{2 \gamma_{x} \mu_{y}}, \quad p_{1}^{\mathrm{TE}}=\frac{-1}{2 \gamma_{x} \mu_{z}}, \quad l_{0}^{\mathrm{TE}}=\frac{k_{0}^{2} \epsilon_{x}}{\gamma_{x}}
$$

$\underline{\text { Case } 2}[\hat{\mathbf{p}}(\omega, \mathbf{k})=-\mathbf{k} \times \alpha \mathbf{k} \times$ and $\hat{\mathbf{q}}(\omega, \mathbf{k})=-\mathbf{k} \times \gamma \mathbf{k} \times]$

TM polarization:

$$
k_{z}^{2}\left(k_{y}\right)=-\frac{1}{2}\left(q_{0}^{\mathrm{TM}}+q_{1}^{\mathrm{TM}}\right)+p_{0}^{\mathrm{TM}} \pm \sqrt{l_{0}^{\mathrm{TM}}+\left(p_{0}^{\mathrm{TM}}+\frac{q_{0}^{\mathrm{TM}}-q_{1}^{\mathrm{TM}}}{2} k_{y}^{2}\right)^{2}}
$$

with

$$
q_{0}^{\mathrm{TM}}=\frac{\epsilon_{y}}{\epsilon_{z}}, \quad q_{1}^{\mathrm{TM}}=1, \quad p_{0}^{\mathrm{TM}}=\frac{-1}{2 \gamma_{x} \mu_{x}}, \quad l_{0}^{\mathrm{TM}}=\frac{k_{0}^{2} \epsilon_{y}}{\gamma_{y}} .
$$

TE polarization:

$$
k_{z}^{2}\left(k_{y}\right)=-\frac{1}{2}\left(q_{0}^{\mathrm{TE}}+q_{1}^{\mathrm{TE}}\right)+p_{0}^{\mathrm{TE}} \pm \sqrt{l_{0}^{\mathrm{TE}}+\left(p_{0}^{\mathrm{TE}}-\frac{q_{0}^{\mathrm{TE}}+q_{1}^{\mathrm{TE}}}{2} k_{y}^{2}\right)^{2}+2 q_{0}^{\mathrm{TE}} p_{1}^{\mathrm{TE}} k_{y}^{2}-q_{0}^{\mathrm{TE}} q_{1}^{\mathrm{TE}} k_{y}^{4}}
$$

with

$$
q_{0}^{\mathrm{TE}}=1, \quad q_{1}^{\mathrm{TE}}=\frac{\gamma_{z}}{\gamma_{y}}, \quad p_{0}^{\mathrm{TE}}=\frac{-1}{2 \gamma_{y} \mu_{y}}, \quad p_{1}^{\mathrm{TE}}=\frac{-1}{2 \gamma_{y} \mu_{z}}, \quad l_{0}^{\mathrm{TE}}=\frac{k_{0}^{2} \epsilon_{x}}{\gamma_{y}}
$$


Case $3[\hat{\mathbf{p}}(\omega, \mathbf{k})=-\mathbf{k} \times \alpha \mathbf{k} \times$ and $\hat{\mathbf{q}}(\omega, \mathbf{k})=-\gamma \mathbf{k} \times \mathbf{k} \times]$

\section{TM polarization:}

$$
k_{z}^{2}\left(k_{y}\right)=-\frac{1}{2}\left(q_{0}^{\mathrm{TM}}+q_{1}^{\mathrm{TM}}\right)+p_{0}^{\mathrm{TM}} \pm \sqrt{l_{0}^{\mathrm{TM}}+\left(p_{0}^{\mathrm{TM}}-\frac{q_{0}^{\mathrm{TM}}+q_{1}^{\mathrm{TM}}}{2} k_{y}^{2}\right)^{2}+2 q_{0}^{\mathrm{TM}} p_{1}^{\mathrm{TM}} k_{y}^{2}-q_{0}^{\mathrm{TM}} q_{1}^{\mathrm{TM}} k_{y}^{4}}
$$

with

$$
q_{0}^{\mathrm{TM}}=\frac{\epsilon_{y} \gamma_{z}}{\epsilon_{z} \gamma_{y}}, \quad q_{1}^{\mathrm{TM}}=1, \quad p_{0}^{\mathrm{TM}}=\frac{-1}{2 \gamma_{y} \mu_{x}}, \quad p_{1}^{\mathrm{TM}}=\frac{-1}{2 \gamma_{z} \mu_{x}}, \quad l_{0}^{\mathrm{TM}}=\frac{k_{0}^{2} \epsilon_{y}}{\gamma_{y}} .
$$

\section{TE polarization:}

$$
k_{z}^{2}\left(k_{y}\right)=-k_{y}^{2}+p_{0}^{\mathrm{TE}} \pm \sqrt{l_{0}^{\mathrm{TE}}+\left(p_{0}^{\mathrm{TE}}\right)^{2}+2\left(p_{1}^{\mathrm{TE}}-p_{0}^{\mathrm{TE}}\right) k_{y}^{2}},
$$

with

$$
p_{0}^{\mathrm{TE}}=\frac{-1}{2 \gamma_{x} \mu_{y}}, \quad p_{1}^{\mathrm{TE}}=\frac{-1}{2 \gamma_{x} \mu_{z}}, \quad l_{0}^{\mathrm{TE}}=\frac{k_{0}^{2} \epsilon_{x}}{\gamma_{x}} .
$$

$\underline{\text { Case } 4}[\hat{\mathbf{p}}(\omega, \mathbf{k})=-\mathbf{k} \times \mathbf{k} \times \alpha$ and $\hat{\mathbf{q}}(\omega, \mathbf{k})=-\mathbf{k} \times \mathbf{k} \times \gamma]$

TM polarization:

$$
k_{z}^{2}\left(k_{y}\right)=-\frac{1}{2}\left(q_{0}^{\mathrm{TM}}+q_{1}^{\mathrm{TM}}\right)+p_{0}^{\mathrm{TM}} \pm \sqrt{l_{0}^{\mathrm{TM}}+\left(p_{0}^{\mathrm{TM}}-\frac{q_{0}^{\mathrm{TM}}+q_{1}^{\mathrm{TM}}}{2} k_{y}^{2}\right)^{2}+2 q_{0}^{\mathrm{TM}} p_{1}^{\mathrm{TM}} k_{y}^{2}-q_{0}^{\mathrm{TM}} q_{1}^{\mathrm{TM}} k_{y}^{4}}
$$

with

$$
q_{0}^{\mathrm{TM}}=\frac{\epsilon_{y}}{\epsilon_{z}}, \quad q_{1}^{\mathrm{TM}}=\frac{\gamma_{z}}{\gamma_{y}}, \quad p_{0}^{\mathrm{TM}}=\frac{-1}{2 \gamma_{y} \mu_{y}}, \quad p_{1}^{\mathrm{TM}}=\frac{-1}{2 \gamma_{y} \mu_{z}}, \quad l_{0}^{\mathrm{TM}}=\frac{k_{0}^{2} \epsilon_{y}}{\gamma_{y}} .
$$

TE polarization:

$$
k_{z}^{2}\left(k_{y}\right)=-k_{y}^{2}+p_{0}^{\mathrm{TE}} \pm \sqrt{l_{0}^{\mathrm{TE}}+\left(p_{0}^{\mathrm{TE}}\right)^{2}}
$$

with

$$
p_{0}^{\mathrm{TM}}=\frac{-1}{2 \gamma_{x} \mu_{x}}, \quad l_{0}^{\mathrm{TM}}=\frac{k_{0}^{2} \epsilon_{x}}{\gamma_{x}} .
$$

Case $5[\hat{\mathbf{p}}(\omega, \mathbf{k})=-\mathbf{k} \times \mathbf{k} \times \alpha$ and $\hat{\mathbf{q}}(\omega, \mathbf{k})=-\mathbf{k} \times \gamma \mathbf{k} \times]$

TM polarization:

$$
k_{z}^{2}\left(k_{y}\right)=-\frac{1}{2}\left(q_{0}^{\mathrm{TM}}+q_{1}^{\mathrm{TM}}\right)+p_{0}^{\mathrm{TM}} \pm \sqrt{l_{0}^{\mathrm{TM}}+\left(p_{0}^{\mathrm{TM}}-\frac{q_{0}^{\mathrm{TM}}+q_{1}^{\mathrm{TM}}}{2} k_{y}^{2}\right)^{2}+2 q_{0}^{\mathrm{TM}} p_{1}^{\mathrm{TM}} k_{y}^{2}-q_{0}^{\mathrm{TM}} q_{1}^{\mathrm{TM}} k_{y}^{4}}
$$

with

$$
q_{0}^{\mathrm{TM}}=\frac{\epsilon_{y}}{\epsilon_{z}}, \quad q_{1}^{\mathrm{TM}}=1, \quad p_{0}^{\mathrm{TM}}=\frac{-1}{2 \gamma_{x} \mu_{y}}, \quad p_{1}^{\mathrm{TM}}=\frac{-1}{2 \gamma_{x} \mu_{z}}, \quad l_{0}^{\mathrm{TM}}=\frac{k_{0}^{2} \epsilon_{y}}{\gamma_{x}} .
$$

TE polarization:

$$
k_{z}^{2}\left(k_{y}\right)=-\frac{1}{2}\left(q_{0}^{\mathrm{TE}}+q_{1}^{\mathrm{TE}}\right) k_{y}^{2}+p_{0}^{\mathrm{TE}} \pm \sqrt{l_{0}^{\mathrm{TE}}+\left(p_{0}^{\mathrm{TE}}+\frac{q_{0}^{\mathrm{TE}}-q_{1}^{\mathrm{TE}}}{2} k_{y}^{2}\right)^{2}},
$$

with

$$
q_{0}^{\mathrm{TE}}=1, \quad q_{1}^{\mathrm{TE}}=\frac{\gamma_{z}}{\gamma_{y}}, \quad p_{0}^{\mathrm{TE}}=\frac{-1}{2 \gamma_{y} \mu_{x}}, \quad l_{0}^{\mathrm{TE}}=\frac{k_{0}^{2} \epsilon_{x}}{\gamma_{y}} .
$$


Case $6[\hat{\mathbf{p}}(\omega, \mathbf{k})=-\mathbf{k} \times \mathbf{k} \times \alpha$ and $\hat{\mathbf{q}}(\omega, \mathbf{k})=-\gamma \mathbf{k} \times \mathbf{k} \times]$

TM polarization:

$$
Q\left(k_{y}\right) k_{z}^{2}\left(k_{y}\right)=P_{0}\left(k_{y}^{2}\right) \pm \sqrt{\left[P_{0}\left(k_{y}\right)\right]^{2}+P_{1}\left(k_{y}^{2}\right)}
$$

where

$$
\begin{aligned}
& Q_{0}\left(k_{y}\right)=2 \delta k_{y}-2 p_{0}^{\mathrm{TM}} \\
& P_{0}\left(k_{y}\right)=-\delta k_{y}^{4}+\left(p_{0}^{\mathrm{TM}}+p_{1}^{\mathrm{TM}}\right) k_{y}^{2}+n_{1} \\
& P_{1}\left(k_{y}\right)=2\left(p_{1}^{\mathrm{TM}} k_{y}^{4}+n_{0} k_{y}^{2}+n_{0} n_{1}\right)\left(2 \delta k_{y}^{2}-2 p_{0}^{\mathrm{TM}}\right)
\end{aligned}
$$

with

$\delta=\left(\gamma_{y}-\gamma_{z}\right)\left(\mu_{y}-\mu_{z}\right), \quad p_{0}^{\mathrm{TM}}=k_{0}^{2} \gamma_{y} \mu_{y} \epsilon_{z} \mu_{z}, \quad p_{1}^{\mathrm{TM}}=k_{0}^{2} \gamma_{z} \mu_{z} \epsilon_{y} \mu_{y}, \quad n_{0}=k_{0}^{2} \epsilon_{y} \mu_{y}, \quad n_{1}=k_{0}^{2} \epsilon_{z} \mu_{z}$.

TE polarization:

$$
k_{z}^{2}\left(k_{y}\right)=-k_{y}^{2}+p_{0}^{\mathrm{TE}} \pm \sqrt{l_{0}^{\mathrm{TE}}+\left(p_{0}^{\mathrm{TE}}\right)^{2}}
$$

with

$$
p_{0}^{\mathrm{TE}}=\frac{-1}{2 \gamma_{x} \mu_{x}}, \quad l_{0}^{\mathrm{TE}}=\frac{k_{0}^{2} \epsilon_{x}}{\gamma_{x}} .
$$

Case $\mathbf{7}[\hat{\mathbf{p}}(\omega, \mathbf{k})=-\alpha \mathbf{k} \times \mathbf{k} \times$ and $\hat{\mathbf{q}}(\omega, \mathbf{k})=-\mathbf{k} \times \mathbf{k} \times \gamma]$

The dispersion relations for both TE and TM polarizations for this case, are identical to Case 4.

Case $8[\hat{\mathbf{p}}(\omega, \mathbf{k})=-\alpha \mathbf{k} \times \mathbf{k} \times$ and $\hat{\mathbf{q}}(\omega, \mathbf{k})=-\mathbf{k} \times \gamma \mathbf{k} \times]$

The dispersion relations for both TE and TM polarizations for this case, are identical to Case 5.

$\underline{\text { Case } \mathbf{9}}[\hat{\mathbf{p}}(\omega, \mathbf{k})=-\alpha \mathbf{k} \times \mathbf{k} \times$ and $\hat{\mathbf{q}}(\omega, \mathbf{k})=-\gamma \mathbf{k} \times \mathbf{k} \times]$

TM polarization:

$$
k_{z}^{2}\left(k_{y}\right)=-\frac{1}{2}\left(q_{0}^{\mathrm{TM}}+q_{1}^{\mathrm{TM}}\right)+p_{0}^{\mathrm{TM}} \pm \sqrt{l_{0}^{\mathrm{TM}}+\left(p_{0}^{\mathrm{TM}}-\frac{q_{0}^{\mathrm{TM}}+q_{1}^{\mathrm{TM}}}{2} k_{y}^{2}\right)^{2}+2 q_{0}^{\mathrm{TM}} p_{1}^{\mathrm{TM}} k_{y}^{2}-q_{0}^{\mathrm{TM}} q_{1}^{\mathrm{TM}} k_{y}^{4}}
$$

with

$$
q_{0}^{\mathrm{TM}}=\frac{\epsilon_{y} \gamma_{z}}{\epsilon_{z} \gamma_{y}}, \quad q_{1}^{\mathrm{TM}}=1, \quad p_{0}^{\mathrm{TM}}=\frac{-1}{2 \gamma_{y} \mu_{y}}, \quad p_{1}^{\mathrm{TM}}=\frac{-1}{2 \gamma_{z} \mu_{z}}, \quad l_{0}^{\mathrm{TM}}=\frac{k_{0}^{2} \epsilon_{x}}{\gamma_{x}} .
$$

TE polarization:

$$
k_{z}^{2}\left(k_{y}\right)=-k_{y}^{2}+p_{0}^{\mathrm{TE}} \pm \sqrt{l_{0}^{\mathrm{TE}}+\left(p_{0}^{\mathrm{TE}}\right)^{2}}
$$

with

$$
p_{0}^{\mathrm{TM}}=\frac{-1}{2 \gamma_{x} \mu_{x}}, \quad l_{0}^{\mathrm{TM}}=\frac{k_{0}^{2} \epsilon_{x}}{\gamma_{x}} .
$$

\section{References}

[1] S. Guenneau and S. A. Ramakrishna, "Negative refractive index, perfect lenses and checkerboards: Trapping and imaging effects in folded optical spaces", Comptes Rendus Physique, vol. 10 (5), p. 352, 2009.

[2] J. B. Pendry, "Negative refraction makes a perfect lens", Physical Review Letters, vol. 85 (18), p. 3966, 2000.

[3] M. G. Silveirinha, A. Alù, and E. Nader, "Infrared and optical invisibility cloak with plasmonic implants based on scattering cancellation", Physical Review B, vol. 78 (7), p. 075107, 2008.

[4] A. Zadpoor, "Design for additive bio-manufacturing: From patient-specific medical devices to rationally designed meta-biomaterials", International Journal of Molecular Sciences, vol. 18 (8), p. 1607, 2017. 
[5] S. A. Ramakrishna, "Physics of negative refractive index materials", Reports on Progress in Physics, vol. 68 (2), p. 449, 2005.

[6] A. Noor, "Metamaterial Electromagnetic Absorbers and Plasmonic Structures", PhD thesis at the University of Manchester (United Kingdom), 2010.

[7] H. Chen, C. T. Chan, and P. Sheng, "Transformation optics and metamaterials", Nature Materials, vol. 9 (5), p. 387, 2010.

[8] J. C. Soric, R. Fleury, A. Monti, A. Toscano, F. Bilotti, and A. Alù, "Controlling Scattering and Absorption With Metamaterial Covers ", IEEE Transactions on Antennas and Propagation, vol. 62, p. 4220, 2014.

[9] C. J. Stevens, A. S. Chu, J. Yan, and E. Shamonina, "Meta-Molecular Devices", 2018 IEEE Radio and Antenna Days of the Indian Ocean (RADIO), 2018.

[10] C. R. Simovski and S. A. Tretyakov, "Local constitutive parameters of metamaterials from an effectivemedium perspective", Physical Review B, vol. 75, p. 195111, 2007.

[11] D. R. Smith and J. B. Pendry, "Homogenization of metamaterials by field averaging", Journal of the Optical Society of America B, vol. 23 (3), p. 391, 2006.

[12] D. R. Smith, S. Schultz, P. Markoš, and C. M. Soukoulis, "Determination of effective permittivity and permeability of metamaterials from reflection and transmission coefficients", Physical Review B, vol. 65 (19), p. 195104, 2002.

[13] C. R. Simovski, "Bloch material parameters of magneto-dielectric metamaterials and the concept of Bloch lattices", Metamaterials, vol. 1 (2), p. 62, 2006.

[14] S. I. Maslovski, S. A. Tretyakov, and C. R. Simovski, "Electromagnetic modelling of composite media with second-order spatial dispersion", XXVIth URSI General Assembly, University of Toronto Canada, vol. 91, 1999.

[15] G. Bouchitté and B. Schweizer, "Homogenization of Maxwell's equations in a split ring geometry", Multiscale Modeling \& Simulation, SIAM, vol. 8(3), p. 717, 2010.

[16] G. Bouchitté, C. Bourel, and D. Felbacq, "Homogenization near resonances and artificial magnetism in three dimensional dielectric metamaterials", Archive for Rational Mechanics and Analysis, vol. 225, p. 1233, 2017.

[17] D. Felbacq and G. Bouchitté, "Homogenization of a set of parallel fibres", Waves in Random Media, vol. 7 (2), p. 245, 1997.

[18] A. Lamacz and B. Schweizer, "Effective Maxwell equations in a geometry with flat rings of arbitrary shape", SIAM Journal on Mathematical Analysis, vol. 45 (3), p. 1460, 2013.

[19] A. Lamacz and B. Schweizer, "A negative index meta-material for Maxwell's equations", SIAM Journal on Mathematical Analysis, vol. 48 (6), p. 4155, 2016.

[20] B. Schweizer, "Resonance meets homogenization", Jahresbericht der Deutschen Mathematiker-Vereinigung, vol. 119 (1), p. 31, 2017.

[21] C. R. Simovski, "On electromagnetic characterization and homogenization of nanostructured metamaterials", Journal of Optics, vol. 13 (1), p. 013001, 2010.

[22] P. Lalanne and D. Lemercier-Lalanne, "On the effective medium theory of subwavelength periodic structures", Journal of Modern Optics, vol. 43 (10), p. 2063, 1996.

[23] M. A. Gorlach and P. A. Belov, "Nonlocality in uniaxially polarizable media", Physical Review B, vol. 92 (8), p. 085107, 2015.

[24] M. G. Silveirinha, "Additional boundary condition for the wire medium", IEEE Transactions on Antennas and Propagation, vol. 54(6), p. 1766, 2006.

[25] M. G. Silveirinha and P. A. Belov, "Spatial dispersion in lattices of split ring resonators with permeability near zero", Physical Review B, vol. 77(23), p. 233104, 2008.

[26] A. Chebykin, A. Orlov, A. Vozianova, S. Maslovski, Y. Kivshar, and P. A. Belov, "Nonlocal effective medium model for multilayered metal-dielectric metamaterials", Physical Review B, vol. 84 (11), p. $115438,2011$. 
[27] A. Ciattoni and C. Rizza, "Nonlocal homogenization theory in metamaterials: effective electromagnetic spatial dispersion and artificial chirality", Physical Review B, vol. 91 (18), p. 184207, 2015.

[28] E. J. C. Dias, D. A. Iranzo, P. Gonçalves, Y. Hajati, Y. V. Bludov, A. P. Jauho, N. A. Mortensen, F. H. L. Koppens, and N. M. R. Peres, "Probing nonlocal effects in metals with graphene plasmons", Physical Review B, vol. 97 (24), p. 245405, 2018.

[29] M. LaBalle and M. Durach, "Additional waves and additional boundary conditions in local quartic metamaterials", OSA Continuum, vol. 2, p. 17, 2019.

[30] K. Mnasri, A. Khrabustovskyi, C. Stohrer, M. Plum, and C. Rockstuhl, "Beyond local effective material properties for metamaterials", Physical Review B, vol. 97, p. 075439, 2018.

[31] V. M. Agranovich and V. Ginzburg, "Spatial Dispersion in Crystal Optics", Springer, p. 136, 1984.

[32] M. G. Silveirinha, J. D. Baena, L. Jelinek, and R. Marques, "Nonlocal homogenization of an array of cubic particles made of resonant rings", Metamaterials, vol. 3 (3-4), p. 115, 2009.

[33] I. Tsukerman, "Nonlocal homogenization of metamaterials by dual interpolation of fields", Journal of the Optical Society of America B, vol. 28 (12), p. 2956, 2011.

[34] A. Serdiukov, I. Semchenko, S. Tertyakov, and A. Sihvola, "Electromagnetics of bi-anisotropic materialsTheory and Application", Gordon and Breach science publishers, vol. 11, p. 2956, 2001.

[35] S. I. Maslovski, T. A. Morgado, M. G. Silveirinha, C. S. R. Kaipa, and A. B. Yakovlev, "Generalized additional boundary conditions for wire media", New Journal of Physics, vol. 12(11), p. 113047, 2010.

[36] M. G. Silveirinha and S. I. Maslovski, "Physical restrictions on the Casimir interaction of metal-dielectric metamaterials: An effective-medium approach", Physical Review A, vol. 82 (5), p. 052508, 2010.

[37] P. Monk, "Finite element methods for Maxwell's equations", Oxford University Press, 2003.

[38] A. Khrabustovskyi, K. Mnasri, M. Plum, C. Stohrer, and C. Rockstuhl, "Interface conditions for a metamaterial with strong spatial dispersion", arXiv:1710.03676v1, 2017.

[39] C. S. R. Kaipa, A. B. Yakovlev, S. I. Maslovski, and M. G. Silveirinha, "Indefinite dielectric response and all-angle negative refraction in a structure with deeply-subwavelength inclusions", Physical Review B, vol. 84(16),p. 165135, 2011.

[40] J. C. Nédéle, "Acoustic and electromagnetic equations: integral representations for harmonic problems", Springer Science \& Business Media, 2001.

[41] K. Mnasri, A. Khrabustovskyi, M. Plum, and C. Rockstuhl, "Retrieving effective material parameters of metamaterials characterized by nonlocal constitutive relations", Physical Review B, vol 99, p. 035442, 2019.

[42] C. Menzel, T. Paul, C. Rockstuhl, T. Pertsch, S. Tretyakov, and F. Lederer, "Validity of effective material parameters for optical fishnet metamaterials", Physical Review B, vol. 81 (3), p. 035320, 2010.

[43] A. Bensoussan, J.-L. Lions and G. Papanicolau, "Asymptotic analysis for periodic structures", NorthHolland Publishing Co., Amsterdam-New York, vol. 5, 1978.

[44] D. Cioranescu, P. Donato, "An Introduction to Homogenization", Oxford University Press, NewYork, 1999.

[45] V. V. Jikov, S. M. Kozlov, and O. A. Oleinik, "Homogenization of differential operators and integral functionals", Springer Science \& Business Media, 2012.

[46] S. Guenneau and F. Zolla, "Homogenization of 3D finite chiral photonic crystals", Physica B: Condensed Matter, vol. 394, pp. 145, 2007.

[47] K. Hiremath, L. Zschiedrich, and F. Schmidt, "Numerical solution of nonlocal hydrodynamic Drude model for arbitrary shaped nano-plasmonic structures using Nédélec finite elements", Journal of Computational Physics, Elsevier, vol 231 (17), p. 5890, 2012.

[48] J. M. McMahon, S. K. Gray, and G. C. Schatz, "Calculating nonlocal optical properties of structureswith arbitrary shape", Physical Review B, vol 82, p. 035423, 2010.

[49] J. Gratus, P. Kinsler, R. Letizia, and T. Boyd, "Electromagnetic mode profile shaping in waveguides", Applied Physics A, vol 123 (1), p. 108, 2017. 
[50] T. Boyd, J. Gratus, P. Kinsler, and R. Letizia, "Customizing longitudinal electric field profiles using spatial dispersion in dielectric wire arrays", Optics Express, vol 26 (3), p. 2478, 2018.

[51] K. Mnasri, F. Z. Goffi, M. Plum, and C. Rockstuhl, "Homogenization of wire media with a general purpose nonlocal constitutive relation", Journal of the Optical Society of America B, vol 36 (8), p. F99, 2019.

[52] T. Geng, S. Zhuang, J. Gao, and X. Yang, "Nonlocal effective medium approximation for metallic nanorod metamaterials", Physical Review B, vol 91 (24), p. 245128, 2015.

[53] L. Li, "New formulation of the Fourier modal method for crossed surface-relief gratings", Journal of the Optical Society of America A, vol 14 (10), p. 2758, 1997.

[54] L. Li, "Fourier modal method for crossed anisotropic gratings with arbitrary permittivity and permeability tensors", Journal of Optics A: Pure and Applied Optics, vol 5(4), p. 345, 2003.

[55] M. Samiullah, "A first course in vibrations and waves", Oxford University Press, 2015.

[56] L. D. Landau and E. M. Lifshitz "Course of theoretical physics 5 - Statistical physics part 1, § 125", Elsevier Science 3rd edition, 2013.

[57] J. D. Jackson "Classical electrodynamics", American Journal of Physics 3rd edition, 1999.

[58] F. G. Friedlander, "Introduction to the Theory of Distributions", Cambridge University Press, 1998.

[59] H. Brezis, "Functional analysis, Sobolev spaces and partial differential equations", Springer Science E6 Business Media, 2010.

[60] V. M. Agranovich and V. Ginzburg, "Crystal optics with spatial dispersion, and excitons", Springer Science Es Business Media, vol. 42, 2013. 